\title{
Adhesion and detachment of a capsule in axisymmetric flow
}

\author{
M. P. Keh and L. G. Leal \\ Department of Chemical Engineering, University of California, Santa Barbara, Santa Barbara, \\ California 93106, USA
}

(Received 22 January 2016; published 9 May 2016)

\begin{abstract}
The adhesion and detachment of a capsule on a solid boundary surface is studied via a combination of scaling theory and numerical simulation and the behavior is compared and contrasted with a vesicle. It is shown that the dominant physical property for both capsules and vesicles is the area dilation modulus $K_{s}$ of the membrane. The nonzero shear modulus $G_{s}$ for capsules increases the resistance to deformation and thus decreases slightly the equilibrium contact radius for an adhered capsule compared to an adhered vesicle. The detachment process in this study is due to an external axisymmetric flow. Unlike a rigid body that must be pulled away without change of shape, capsules (and vesicles) almost always detach dominantly by peeling in which the contact radius decreases but the minimum separation distance does not change until the final moments of detachment. Compared to a vesicle with the same $K_{s}$, a capsule maintains a more compact shape and is harder to elongate under a given external flow. Hence, the detachment process is slower for capsules compared to vesicles with the same $K_{s}$.
\end{abstract}

DOI: 10.1103/PhysRevFluids.1.013201

\section{INTRODUCTION}

Capsules and vesicles are particles consisting of a liquid core enclosed within a thin membrane. Such particles are widely used in many industrial applications such as personal care, food, and pharmaceutical products and have also been used as simplified models for biological cells [1-3]. These membrane bound particles are often designed to deliver active ingredients to target surfaces. For example, capsules are used to deliver fragrance oils in shampoo and fabric conditioning products to hair and clothes. Therefore, adhesion and retention of a capsule or vesicle on a surface is crucial to product effectiveness. Furthermore, adhesion of two capsules or vesicles represents the initial step in the formation of multiparticle clusters that can affect the stability of the product suspension. In many of these applications, soft particles are pushed towards a target surface by an external flow.

In general, there are three important stages in the overall process of adhesion and detachment between a soft particle and a stationary boundary, or between two particles. The first stage involves the hydrodynamic drainage of suspending fluid from between the two surfaces. In this stage, hydrodynamic forces dominate over surface forces because the range of hydrodynamic forces scales as the size of the particle (usually on the order of micrometers) while the range of surface forces (e.g., van der Waals, electrostatic, dispersion, or depletion forces) is a few nanometers. Whatever its initial shape, the capsule or vesicle may deform during this first stage as a consequence of hydrodynamic forces working against the elasticity of the membrane. Once the two surfaces pass through the first stage and get close enough, attractive surface forces take over and pull the particle into an energy well and also cause the membrane to deform and spread along the adhesive surface. We will refer to the capture of a particle in an energy well near the surface as adhesion. Finally, in the third stage, the flow could change direction and attempt to detach the adhered particle. The hydrodynamic thin-film drainage stage has already been studied thoroughly by Frostad et al. [4] and Keh et al. [5] for both vesicles and capsules. In the present work we start by investigating the equilibrium adhesion configuration with a given combination of surface forces in the absence of any external flow and then use the equilibrium configuration as the initial condition to study the dynamics of detachment under flow. We then further study detachment of an adhered capsule starting from nonequilibrium configurations where the capsule is not given enough time to equilibriate with the surface forces. 
The equilibrium contact radius has been studied extensively for both inextensible (bending modulus dominated) and extensible (area dilation modulus dominated) vesicles [6-9]. Explicit formula and scaling relations for the equilibrium contact radius as a function of adhesion strength and membrane modulus have been derived. What makes a capsule membrane different from a vesicle membrane is its resistance to in-plane shear due to the fact that the surface shear modulus is nonzero. When it comes to capsules, there are a number of investigations that study capsule-substrate adhesion [10-12]. However, there is still ambiguity about how important the surface shear modulus is as researchers often disallow in-plane shear or adopt the widely used neo-Hookean model that couples the surface shear modulus and area dilation modulus together. This prevents them from investigating the relative importance of the two modes of deformation, i.e., in-plane shear and isotropic stretching, in the adhesion stage. In this study we are looking at cases with a short-range repulsive surface interaction (electrostatic or steric) and a long-range attractive interaction (depletion or hydrophobic). Adhesive contact in such cases means the membrane sits and spreads along the interaction potential minima constructed by both the short-range and long-range surface interactions with a thin fluid film remaining in-between the membrane and the solid surface.

The dynamics of detachment of an adhered membrane-bound particle has received increasing interest in recent years [13-16]. The classical work of Brochard-Wyart and de Gennes [13], which considers the detachment of a vesicle that is partially aspirated on one side and stuck on the other side to a flat plate, provides a good theoretical starting point. This particular configuration significantly reduces the complexity of the problem since the tension on the vesicle membrane is uniform and the magnitude of the tension is fixed by the pipette suction pressure. These simplifications need to be lifted when capsules, instead of vesicles, are considered or other types of external driving force for detachment such as flow or gravity are considered. Furthermore, recent experimental studies [15,16] showed that, in some cases, the measured force and rate of detachment are drastically different from what the existing theory would predict. There is clearly a need to investigate the source of the discrepancy between the existing theory and experiments and develop a more general understanding. There is also a debate regarding the conditions when the pulling mechanism (i.e., increase in the width of the thin film, as occurs for a rigid body) will dominate over the peeling mechanism (i.e., decrease in the contact radius with no change in the film thickness) during the detachment process [16].

We present simulation results from a numerical model, based on the work of Walter et al. [17], coupling the boundary integral method for the motion of the fluids, a finite-element method for the membrane mechanics, and the Derjaguin approximation for the surface forces acting between the membrane and the solid boundary. Most of our focus will be on initially stress-free capsules with more limited results on initially stress-free vesicles for comparison. In general, we assume that the membrane thickness is very small compared to the size of the particle and thus the bending stiffness is negligible. The detailed equations and constitutive laws that we use to model the problem will be introduced in Sec. II. In Sec. III we first report numerical results for the equilibrium contact radius of capsules as a function of adhesion energy per unit area, surface shear modulus, and area dilation modulus. These results are then compared to numerical and theoretical results for vesicles to illustrate any differences introduced by the additional in-plane shear resistance of capsules. We demonstrate that the pulling mechanism only matters at the very end of the detachment process, whereas the peeling process dominates almost the entire process and determines the maximum dynamic force measured during detachment. A more general theory for the peeling velocity as a function of adhesion strength and flow strength is developed based on an overall energy balance and this is corroborated by detailed numerical simulations. Finally, the force required to peel off an adhered particle at a given peeling velocity is also extracted from the theory and this is compared to the forces required to separate two vesicles, as measured by Frostad et al. [16].

\section{PROBLEM STATEMENT AND METHODS}

We consider the hydrodynamic and nonhydrodynamic interactions between a membrane-bound particle (i.e., either a capsule or a vesicle) and a rigid and stationary sphere (the collector) of radius 


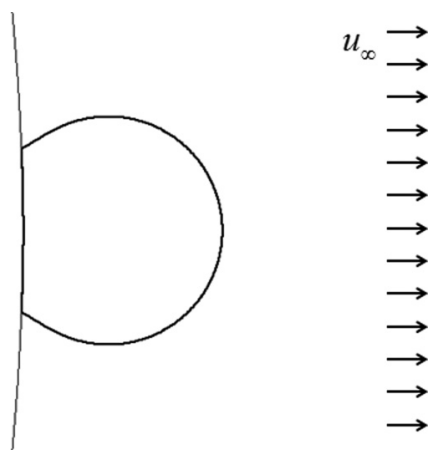

FIG. 1. Geometry of a capsule with undeformed radius $R_{0}$ adhered to a stationary solid sphere of radius $R_{s}=20 R_{0}$ with an unperturbed uniform flow $u_{\infty}$ trying to detach the capsule.

$R_{s}$. We assume that the particle is spherical with radius $R_{0}$ that is much smaller than $R_{s}(1: 20)$. First, the particle is placed in the vicinity of the rigid sphere, within the range of nonhydrodynamic forces, and the membrane is allowed to deform and reach an equilibrium configuration with a contact radius of $a_{\text {eq }}$. The far-field uniform flow $u_{\infty}$ is then turned on in an attempt to detach the adhered particle and the dynamic process of detachment is observed and studied (see Fig. 1). In simulation, we are mainly looking at capsules, with some supplementary vesicle cases. In the present section we outline the equations governing the problem and the method used to study it numerically. The method of this study largely follows the preceding work of Keh et al. [5] with nonhydrodynamic surface interactions incorporated into it. Note that we choose to use a spherical collector that is much larger than the capsule instead of a flat wall because the spherical geometry significantly simplifies the boundary integral equation and enables future investigation of the effect of the collector curvature.

\section{A. Nonhydrodynamic surface interactions}

An adhesive energy well is composed of a long-range attractive force and a short-range repulsive force. In numerical simulations, we construct the energy well with a certain depth $\left|W_{0}\right|$ and a location $D_{0}$ for the energy minimum using combinations of electrostatic repulsive force and either hydrophobic attraction or polymer depletion attraction forces. The forces are calculated analytically using the Derjaguin approximation, which approximates the interaction between a small planar membrane element and the collector surface as that between the small element and an infinite plane. The three types of surface forces per unit area can be expressed as follows $[18,19]$ :

$$
\begin{gathered}
f_{\text {electrostatic }}(D)=\frac{\kappa^{2}}{2 \pi} Z \exp ^{-\kappa D}, \\
f_{\text {hydrophobic }}(D)=-2 \frac{\gamma}{D_{\text {hydro }}} \exp ^{-D / D_{\text {hydro }},} \\
f_{\text {depletion }}(D)=-\rho_{p} k_{B} T \mathrm{H}\left(R_{g}-D\right) .
\end{gathered}
$$

Here $D$ is the separation between the membrane element and the rigid surface, $\kappa$ and $Z=9.22 \times$ $10^{-11} \tanh ^{2}\left(\psi_{0} / 103\right)$ are the inverse of the Debye length and the electrostatic interaction constant at $298 \mathrm{~K}$ in 1:1 electrolyte solutions (where $\psi_{0}$ is the surface potential in units of $\mathrm{mV}$ ), respectively, $\gamma$ is the interfacial energy per unit area, $D_{\text {hydro }}=1 \mathrm{~nm}$ is the characteristic length scale of the hydrophobic interaction, $\rho_{p}$ is the number density of the nonadsorbing polymer chains in the bulk solution, $k_{B}$ is the Boltzmann constant, $T$ is the absolute temperature, $\mathrm{H}$ represents the Heaviside function, and $R_{g}$ is the radius of gyration of the polymer. The associated surface interaction energies 
per unit area $W_{\text {electrostatic }}, W_{\text {hydrophobic }}$, and $W_{\text {depletion }}$ can be found by integrating Eqs. (1)-(3) with respect to $D$, respectively. Since we focus on the dynamics of adhesion and detachment, we assume that the membrane surface and the rigid surface are identical in terms of chemical properties and the energy well is constructed using one repulsive and one attractive surface interaction, either electrostatic with hydrophobic attraction or electrostatic with depletion attraction, to simplify the nonhydrodynamic part of the problem. Also note that in the following discussion, only $\left|W_{0}\right|$ and $D_{0}$ will be given with all other secondary parameters such as $\psi_{0}$ and $\kappa$ shielded from the reader since they are not essential to our analysis. The load on the membrane due to nonhydrodynamic interactions (in the absence of flow) $\boldsymbol{f}_{\text {int }}$ can be found:

$$
\boldsymbol{f}_{\text {int }}(\boldsymbol{x})=\left[f_{\text {electrostatic }}(D(\boldsymbol{x}))+f_{\text {hydrophobic }}(D(\boldsymbol{x}))+f_{\text {depletion }}(D(\boldsymbol{x}))\right] \cos (\theta) \boldsymbol{n},
$$

where $\boldsymbol{n}$ is the outward-pointing unit normal of the surface on which the load is exerted and $\theta$ is the angle between $\boldsymbol{n}$ and the vector normal to the hypothetical infinite plane.

\section{B. Hydrodynamics}

The fluid inside and outside the membrane is Newtonian and the Reynolds number of the flow based on the size of the capsule or vesicle is assumed to be small enough that the hydrodynamics of the problem is governed by the Stokes flow equations

$$
\begin{gathered}
\mu \nabla^{2} \boldsymbol{u}-\nabla p=0, \\
\nabla \cdot \boldsymbol{u}=0,
\end{gathered}
$$

where $\mu$ is the viscosity, $\boldsymbol{u}$ is the velocity, and $p$ is the pressure in the external flow. The same equations are valid inside the membrane, using the denotations $\hat{\mu}, \hat{\boldsymbol{u}}$, and $\hat{p}$ for the internal viscosity, velocity field, and pressure, respectively. The no-slip and kinematic conditions are applied at the two surfaces: the capsule or vesicle membrane $S_{1}$ and the rigid surface of the collector $S_{2}$ :

$$
\begin{gathered}
\boldsymbol{u}(\boldsymbol{x})=\hat{\boldsymbol{u}}(\boldsymbol{x}), \quad \boldsymbol{x} \in S_{1} \\
\boldsymbol{u}(\boldsymbol{x})=0, \quad \boldsymbol{x} \in S_{2} .
\end{gathered}
$$

We assume the internal viscosity is equal to the external viscosity $\hat{\mu}=\mu$ for all the analysis, which greatly simplifies the boundary integral equations. Our expectation is that this case should be representative for systems in which the internal viscosity is the same order of magnitude as or smaller than the exterior viscosity. The exception occurs when the internal viscosity is much larger, thus rendering the capsule or vesicle as largely undeformable on the time scales of the collision process. However, the problem of a solid particle approaching and adhering to a solid wall has already been well studied [20,21] and is outside the scope of this study.

Writing the weak form of the previous equations, the hydrodynamic problem can be rewritten as boundary integral equations on $S_{1}$ and $S_{2}$ (see, e.g., Ref. [22]):

$$
\boldsymbol{u}(\boldsymbol{x})=\boldsymbol{u}_{0}(\boldsymbol{x})-\frac{1}{8 \pi \mu} \int_{S_{1} \cup S_{2}} \boldsymbol{J}(\boldsymbol{x}, \boldsymbol{y}) \cdot \boldsymbol{f}(\boldsymbol{y}) d A(\boldsymbol{y}) \forall \boldsymbol{x} \in S_{1} .
$$

Here the Oseen tensor is

$$
\boldsymbol{J}(\boldsymbol{x}, \boldsymbol{y})=\frac{1}{\tilde{r}} \boldsymbol{I}+\frac{1}{\tilde{r}^{3}} \tilde{\boldsymbol{r}} \otimes \tilde{\boldsymbol{r}},
$$

where $\tilde{\boldsymbol{r}}=\boldsymbol{x}-\boldsymbol{y}$ and $\tilde{\boldsymbol{r}}=\|\tilde{\boldsymbol{r}}\|$. The velocity $\boldsymbol{u}_{0}$ is the unperturbed velocity field (without the particle and the collector). For the investigation of the equilibrium contact radius in the absence of ambient flow, we set $\boldsymbol{u}_{0}(\boldsymbol{x})=0$. For the detachment study, we consider a uniform ambient flow

$$
\boldsymbol{u}_{0}(\boldsymbol{x})=u_{\infty} \boldsymbol{e}_{z} .
$$


The load on the membrane due to flow (in the absence of nonhydrodynamic interactions) $\boldsymbol{f}_{\text {dyn }}$ corresponds to the viscous traction jump on $S_{1}$ and the viscous traction on $S_{2}$ :

$$
\begin{gathered}
\boldsymbol{f}_{\mathrm{dyn}}(\boldsymbol{x})=[\boldsymbol{\sigma}-\hat{\boldsymbol{\sigma}}] \cdot \boldsymbol{n}, \quad \boldsymbol{x} \in S_{1} \\
\boldsymbol{f}_{\mathrm{dyn}}(\boldsymbol{x})=\boldsymbol{\sigma} \cdot \boldsymbol{n}, \quad \boldsymbol{x} \in S_{2},
\end{gathered}
$$

where $\boldsymbol{\sigma}$ and $\hat{\boldsymbol{\sigma}}$ are the stress tensors in the external and internal fluids, respectively, and $\boldsymbol{n}$ is the outward-pointing normal.

On the membrane the force can be obtained from the mechanics of the membrane, but it is unknown on the rigid wall. It is however possible to write the boundary integral equation on $S_{2}$,

$$
0=\boldsymbol{u}_{0}(\boldsymbol{x})-\frac{1}{8 \pi \mu} \int_{S_{1} \cup S_{2}} \boldsymbol{J}(\boldsymbol{x}, \boldsymbol{y}) \cdot \boldsymbol{f}_{\mathrm{dyn}}(\boldsymbol{y}) d A(\boldsymbol{y}) \forall \boldsymbol{x} \in S_{2},
$$

from which $\boldsymbol{f}_{\text {dyn }}$ on $S_{2}$ may be obtained.

In an axisymmetric geometry, the boundary integral equations can be preintegrated along the orthoradial direction and rewritten as line integrals with a suitable expression for the Oseen tensor $\boldsymbol{J}$. The expression, which uses elliptic integrals, can be found in Ref. [22].

\section{Mechanics of the membrane}

We treat the membrane of the capsule or vesicle as a bidimensional piece of isotropic hyperelastic material with negligible bending stiffness. This is now a classical approach for a capsule or vesicle with a thin membrane compared to its radius; the details of the mechanical treatment may be found in, e.g., Ref. [23] and we only give here the key equations. The local strain is defined by considering the patch of membrane surrounding a material point $\boldsymbol{X}$ in the unstressed reference state, which becomes $\boldsymbol{x}$ in the deformed state. The gradient of the transformation is then $\boldsymbol{f}=\partial \boldsymbol{x} / \partial \boldsymbol{X}$ and the Cauchy-Green dilation tensor is $\boldsymbol{C}=\boldsymbol{f}^{T} \cdot \boldsymbol{f}$. It is postulated that there exists a free energy per unit undeformed area $w_{s}$ that varies with $\boldsymbol{C}$ and is minimal in the reference state. It can then be shown that the Cauchy tension $\boldsymbol{T}$ in the deformed state takes the form

$$
\boldsymbol{T}=\frac{2}{J_{s}} \frac{\partial w_{s}}{\partial I_{1}} \boldsymbol{f} \cdot \boldsymbol{f}^{T}+2 J_{s} \frac{\partial w_{s}}{\partial I_{2}} \boldsymbol{I}_{s}
$$

where $J_{s}=\operatorname{det} \boldsymbol{f}, \boldsymbol{I}_{s}$ is the bidimensional identity tensor tangential to the surface, and the Skalak invariants $I_{1}$ and $I_{2}$ are [24]

$$
I_{1}=\operatorname{Tr} C-2, \quad I_{2}=\operatorname{det} C-1 .
$$

Several expressions for $w_{s}$ have been proposed to describe the physics of the capsule membrane elasticity. In this study we use Skalak's law [24]

$$
w_{s}^{S k}=\frac{G_{s}}{8}\left(2 I_{1}^{2}+4 I_{1}-4 I_{2}-I_{2}^{2}\right)+\frac{K_{s}}{8} I_{2}^{2},
$$

where $G_{s}$ and $K_{s}$ are the membrane's small-deformation surface shear and area dilation moduli, respectively. We choose Skalak's law over the popular neo-Hookean law because the two moduli are independent in Skalak's law, which allows us to probe the relative importance of $K_{s}$ and $G_{s}$. Note, however, that $K_{s}<G_{s}$ corresponds to a negative surface Poisson ratio, which is a rare material behavior and will not be considered in this study. For comparison, we also consider unilamellar fluidlike vesicles in the linear regime

$$
w_{s}=\frac{K_{s}}{2}\left(J_{s}-1\right)
$$

This law leads to an isotropic tension proportional to the relative area increase $\boldsymbol{T}=K_{s} J_{s} \boldsymbol{I}_{s}$. 
Considering that the inertia of the membrane is negligible compared to the stresses caused by the viscous flow and surface forces, the local equilibrium of a patch of membrane reads

$$
\nabla_{s} \cdot \boldsymbol{T}+\boldsymbol{f}_{\mathrm{ext}}=0
$$

where $\nabla_{s}$ is the surface divergence operator and $\boldsymbol{f}_{\text {ext }}=\boldsymbol{f}_{\text {dyn }}+\boldsymbol{f}_{\text {int }}$ is the total force exerted on the membrane by the flow and surface interactions. Introducing a virtual velocity field $\tilde{\boldsymbol{v}}$ and the corresponding virtual rate of strain tensor $\tilde{\boldsymbol{E}}=\left(\nabla_{s} \tilde{\boldsymbol{v}}+\nabla_{s} \tilde{\boldsymbol{v}}^{T}\right) / 2$, Eq. (19) can be rewritten in variational form showing the balance of the virtual rates of work by the internal and external forces:

$$
\int_{S_{1}} \boldsymbol{T}: \tilde{\boldsymbol{E}} d A=\int_{S_{1}} \boldsymbol{f}_{\mathrm{ext}} \cdot \tilde{\boldsymbol{v}} d A \forall \tilde{\boldsymbol{v}} .
$$

\section{Numerical method}

We first study the equilibrium shape of the adhered capsule without any imposed external flow. This calculation is independent of the initial position or whether the capsule is predeformed by either a flow or external forces before the surface interactions between the capsule and the collector come into play. We choose to solve it as a transient problem starting from a spherical capsule of radius $R_{0}$ that is placed on the axis of symmetry $\boldsymbol{e}_{z}$ at a minimum surface separation $D_{\text {axis }}$ from the spherical collector. The solution up to equilibrium depends on the arbitrarily chosen initial positions and deformed shape, but the final steady-state result does not. We then calculate the dynamical process by which the capsule or vesicle may be detached starting from the previously calculated equilibrium configuration. The full transient solution is of interest in this part of the calculation. Detachment starting from a nonequilibrium initial condition is considered in Sec. III E.

We use an axisymmetric variation of the boundary integral - finite-element coupling strategy developed by Walter et al. [17]. We refer to that paper for details of the numerical method and only give here the general framework. Because the problem is expressed only through equations over the membrane $S_{1}$ and the rigid collector $S_{2}$, only these two surfaces need to be meshed. This is done using quadratic (three-node) elements. Initially, a spherical capsule of radius $R_{0}$ is placed on the axis of symmetry $\boldsymbol{e}_{z}$ at a minimum surface separation $D_{\text {axis }}$ from the spherical collector and the surface forces and/or the flow is turned on. Later on, at any given time step, the shape of the capsule is known. The total force $f_{\text {ext }}$ exerted by the flow and the surface forces on the membrane $S_{1}$ can then be obtained by solving Eq. (20) using the finite-element method. The surface interaction force $\boldsymbol{f}_{\text {int }}$, and therefore the dynamic force $\boldsymbol{f}_{\mathrm{dyn}}=\boldsymbol{f}_{\text {ext }}-\boldsymbol{f}_{\text {int }}$, on the membrane $S_{1}$ can be obtained by Eq. (4). The dynamic force $f_{\text {dyn }}$ on the collector $S_{2}$ is unknown, but can be obtained from Eq. (14), as described by, e.g., Quéguiner and Barthès-Biesel [25]. Then the boundary integral equation (9) can be used explicitly to compute the velocities of the nodes of $S_{1}$. The position of the membrane at the next time step is then obtained through an explicit Euler integration scheme

$$
\boldsymbol{x}(t+\Delta t)=\boldsymbol{x}(t)+\boldsymbol{u}(\boldsymbol{x}, t) \Delta t .
$$

Note that the nonhydrodynamic surface interaction force $f_{\text {int }}$ on the collector surface $S_{2}$ does not play any role in the hydrodynamic problem since the collector is rigid and stationary.

It is well established when using the boundary integral method with thin films that care must be taken that the interfacial node spacing in the thin-film region decreases proportionally with the film thickness. Therefore, the surfaces are regularly remeshed to ensure that the spacing between two nodes is never greater than 1.3 times the distance to the nearest node on the opposite surface. For the numerical method of Walter et al. [17], the time step $\Delta t$ has to decrease proportionally with the size of the smallest membrane element; this is enforced when remeshing.

Initially, the surfaces $S_{1}$ and $S_{2}$ use a nonuniform mesh with element sizes ranging between 0.001 and 0.1 times the initial radius of the particle $R_{0}$ and the initial time step is $\left(G_{s} / \mu R_{0}\right) \Delta t=0.01$. For each time step and each element, we compute the ratio of the distance to the nearest element on the other surface and the mesh size. If the ratio is smaller than 1.3 for any of the elements, 
then all elements for which the ratio is smaller than 1.5 are cut into half and the size of the time step is divided by 2 . Since the internal volume of the capsule is conserved for all our cases, we checked after every time step that the accumulated internal volume change, compared to the initial internal volume, is less than $1 \%$. Numerical integration is generally performed using a classical six-point Gauss-Legendre quadrature method. However, when points $\boldsymbol{x}$ and $\boldsymbol{y}$ become close in the axisymmetric versions of Eqs. (9) and (14), the integrand takes on a logarithmic behavior for which Gauss-Legendre points are ill suited; in that case, the ten-point scheme devised by Smith [26] is used instead.

\section{SCALING THEORIES AND SIMULATION RESULTS}

\section{A. Equilibrium contact radius}

When there is a potential energy well with depth $\left|W_{0}\right|$ at surface separation $D_{0}$ between the membrane surface and the solid surface, it is favorable for the membrane to spread and increase the contact radius $a$ along the solid surface at separation $D_{0}$ as spreading reduces the surface interaction energy. However, the spreading results in an increase in the degree of deformation and the elastic energy. The equilibrium contact radius $a=a_{\text {eq }}$ is reached when the sum of the interaction energy $U_{\text {interaction }}$ and the elastic energy $U_{\text {elastic }}$ is a minimum. The interaction energy can be approximated by the contact area times the adhesion strength $\left|W_{0}\right|$ :

$$
U_{\text {interaction }} \approx-\left|W_{0}\right| \pi a^{2} .
$$

For a vesicle, the elastic energy is equal to the area dilation energy and can be approximated based on conservation of the internal volume assuming a truncated sphere geometry

$$
U_{\text {elastic }}=U_{\text {area }}=K_{s} \frac{\left(A-A_{0}\right)^{2}}{A_{0}} \approx \frac{1}{256} K_{s}\left(\frac{a}{R_{0}}\right)^{8} A_{0} .
$$

Here $A$ is the surface area of the membrane under deformation and $A_{0}=4 \pi R_{0}^{2}$ is the initial surface area. The detailed derivation of Eq. (23) can be found in Sec. 2.1 of Ref. [9]. The lack of a shear penalty results in a uniform and isotropic tension on a deformed vesicle membrane since a vesicle membrane can adjust its configuration freely to distribute the area strain evenly and thus minimize the total elastic energy. There could be slight nonuniformities in tension to account for the varying amount of local tangential stress as shown by Frostad et al. [4]. For a capsule membrane, however, there is a surface shear energy term $U_{\text {shear }}$ in addition to $U_{\text {area }}$ and the tension is expected to be nonuniform and nonisotropic. Unlike the surface area dilation term, the shear term is essentially impossible to estimate from the overall deformed shape of the membrane because shear deformation is often highly localized and nonisotropic.

We will turn to simulations to investigate the surface shear energy later. For now, we are going to assume that $U_{\text {shear }}$ is negligible compare to $U_{\text {area }}$ for adhered capsules due to the fact that the deformation is mostly an isotropic expansion except near the edge of the contact region. The equilibrium contact radius can then be derived for both vesicles and capsules by minimization of the total energy [the sum of Eqs. (22) and (23)]

$$
\begin{gathered}
\frac{d}{d a}\left(U_{\text {interaction }}+U_{\text {elastic }}\right)=0 \quad \text { at } a=a_{\mathrm{eq}}, \\
\frac{a_{\mathrm{eq}}}{R_{0}}=\left(\frac{16\left|W_{0}\right|}{K_{s}}\right)^{1 / 6} .
\end{gathered}
$$

The expectation is that Eq. (25) should work well for vesicles, but will slightly overestimate $a_{\text {eq }}$ for capsules due to the fact that Eq. (25) neglects a small part of the elastic energy penalty that is coming from shear deformation. We will check our assumptions and expectations with simulation 

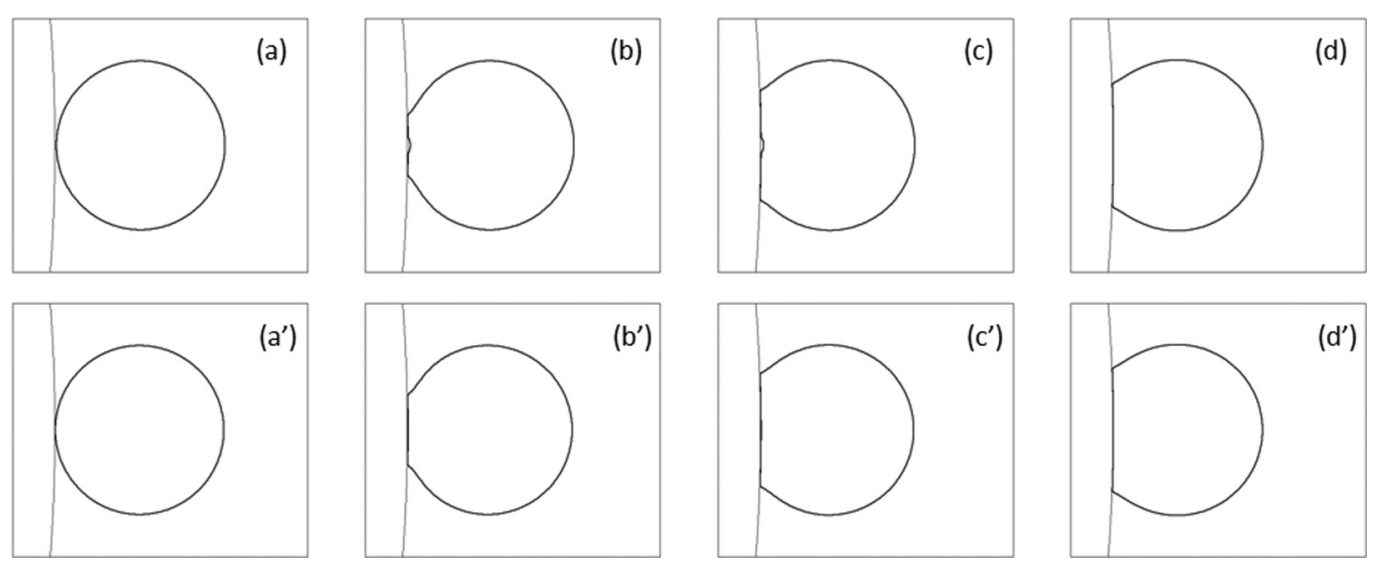

FIG. 2. Spreading process of a capsule near an adhesive rigid boundary at $\left|W_{0}\right| / K_{s}=0.017, K_{s} / G_{s}=3$, and $u_{\infty}=0$ from simulation. The initial surface separation is equal to (a)-(d) $D_{\text {axis }}=3 D_{0}$ and $\left(\mathrm{a}^{\prime}\right)-\left(\mathrm{d}^{\prime}\right)$ $D_{\text {axis }}=D_{0}$. From left to right the dimensionless time evolution is $\tau=t K_{s} / \mu R=0,600,1800$, and 6000 .

results. Note that this equilibrium shape is unique for each $\left|W_{0}\right|$ because the surface interaction potentials used do not have multiple minima.

In simulations, a vesicle or a capsule is initially placed near the collector within the range of the surface forces and is given sufficient time to deform and reach the equilibrium configuration with zero far-field flow $u_{\infty}=0$. Figure 2 shows the spreading process of a capsule for two different initial surface separations. If the front of the membrane is started some distance away from the location of the energy well (for example, $D_{\text {axis }}=3 D_{0}$ ), a dimple is likely to form while the whole particle moves towards the rigid surface as shown in Figs. 2(a)-2(d). This results in some trapped suspending fluid in the middle of the contact area that needs to be drained before reaching the

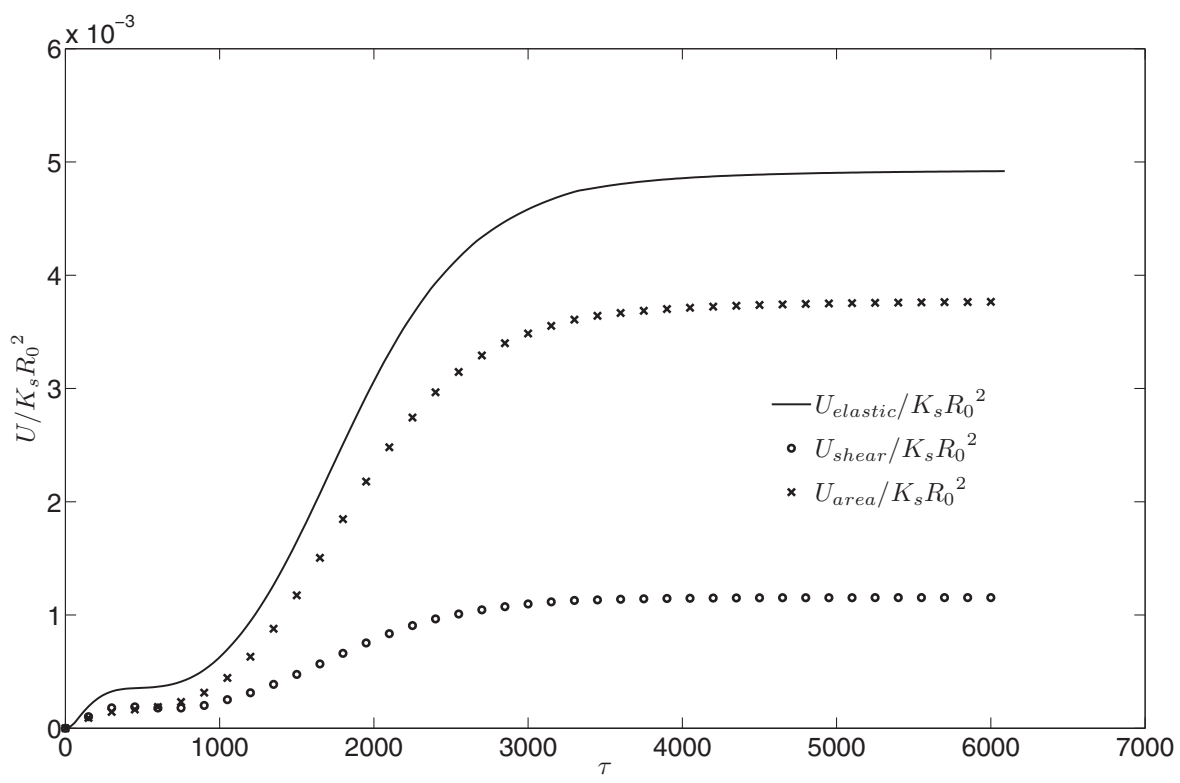

FIG. 3. Dimensionless elastic energy and its components (shear and area dilation) corresponding to the cases of Figs. $2\left(a^{\prime}\right)-2\left(d^{\prime}\right)$ as a function of dimensionless time. 


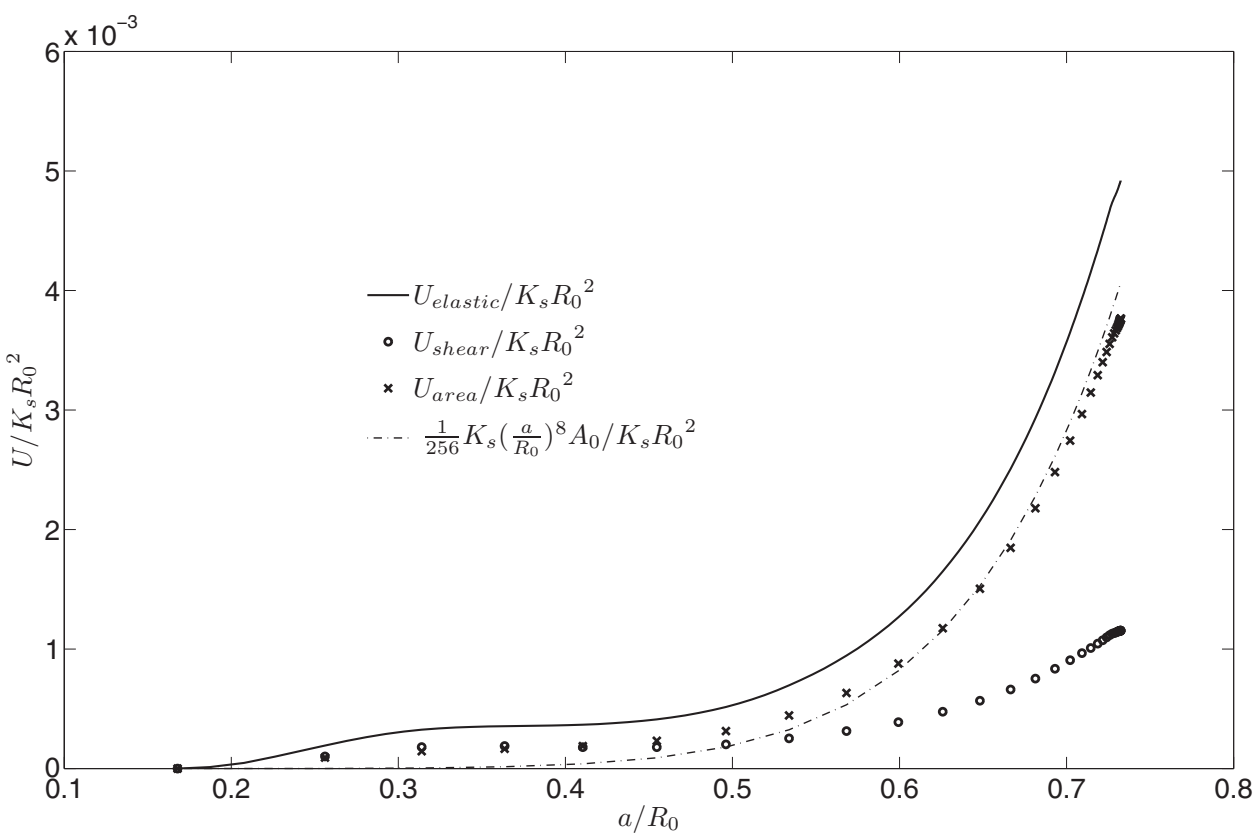

FIG. 4. Dimensionless elastic energy and its components (shear and area dilation) corresponding to the cases of Figs. $2\left(\mathrm{a}^{\prime}\right)-2\left(\mathrm{~d}^{\prime}\right)$ as a function of dimensionless contact radius.

equilibrium configuration. However, during this process, there is minimal change in the contact radius. The dimple can be prevented by placing the front of the membrane close to or right on the energy well $\left(D_{\text {axis }}=D_{0}\right)$ as shown in Figs. $2\left(\mathrm{a}^{\prime}\right)-2\left(\mathrm{~d}^{\prime}\right)$.

The initial surface separation between the membrane and the rigid surface affects the dynamics of the spreading process but has no influence on the equilibrium contact radius. For a capsule, surface area dilation energy and surface shear energy go up due to the increase in degree of deformation as the contact radius increases and approaches the equilibrium value. A typical pattern of $U_{\text {area }}$ and $U_{\text {shear }}$ as a function of time and contact radius for a capsule during the spreading process is shown in Figs. 3 and 4. In all the cases that we have studied numerically, with $a_{\mathrm{eq}} / R_{0}$ ranging from 0.389 to 0.732 and $K_{s} / G_{s}$ ranging from 1.1 to 7 , the elastic energy is dominated by its area dilation component and most importantly $U_{\text {area }}$ increases much faster than $U_{\text {shear }}$ as the contact radius goes up. Furthermore, Eq. (23) is found to be an excellent approximation for $U_{\text {area }}$ except at the early stages of spreading where the deformation on the membrane is highly localized at the front of the membrane that is close to the rigid surface. These observations justify the assumption that $U_{\text {shear }}$ can be neglected in the derivation of the scaling relation for $a_{\mathrm{eq}}$ and Eq. (25) should be applicable to both vesicles and capsules.

Figure 5 confirms that Eq. (25) works well for vesicles, but slightly overestimates $a_{\text {eq }}$ for capsules. Nevertheless, the scaling relation $a_{\text {eq }} / R_{0} \sim\left(\left|W_{0}\right| / K_{s}\right)^{1 / 6}$ holds for capsules. The neglected shear modulus effect can be compensated for by lowering the coefficient from 16 to 11.43 in Eq. (25) or equivalently for capsules

$$
U_{\text {elastic }}=c_{1} \frac{1}{256} K_{s}\left(\frac{a}{R_{0}}\right)^{8} A_{0},
$$

with $c_{1} \approx 1.4$ for cases with $K_{s} / G_{s}=3$. Note that $c_{1}$ should approach unity as $K_{s} / G_{s}$ goes to infinity or $c_{1}=1$ for zero shear modulus (vesicles). In Fig. $6, a_{\mathrm{eq}} / R_{0}$ is shown to be insensitive to $\left|W_{0}\right| / G_{s}$ while holding $\left|W_{0}\right| / K_{s}$ at a fixed value, which serves as further evidence to our claim that $G_{s}$ is only of secondary importance. 


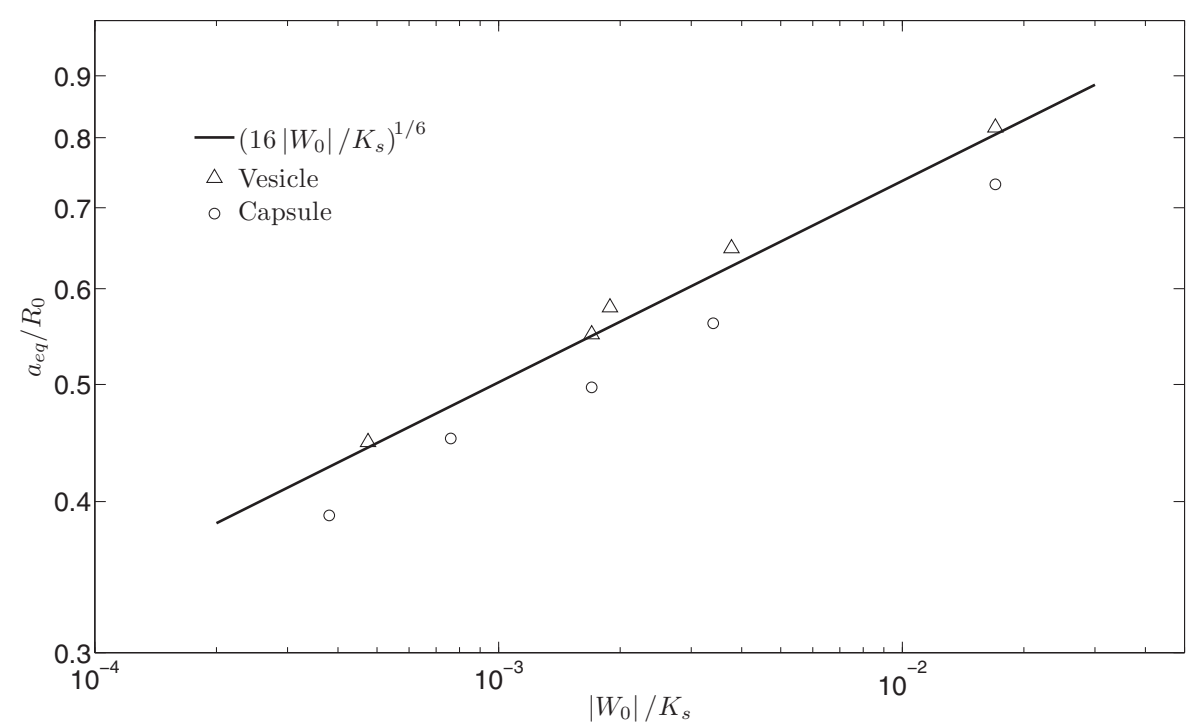

FIG. 5. Dimensionless equilibrium contact radius of vesicles and capsules versus relative adhesion strength based on the surface area dilation modulus $\left|W_{0}\right| / K_{s}$ at a fixed ratio of the two moduli $K_{s} / G_{s}=3$ (capsules only).

\section{B. Detachment mechanism: Peeling, pulling, and critical detachment capillary number}

Once the equilibrium contact radius is established, the far-field uniform flow is turned on in an attempt to detach the adhered particle. During the detachment process, the contact radius decreases from $a_{\text {eq }}$ to zero and the surface separation on the line of centers $D_{\text {axis }}$ increases from $D_{0}$ over time. These two mechanism are referred to as peeling and pulling, respectively. It was well established

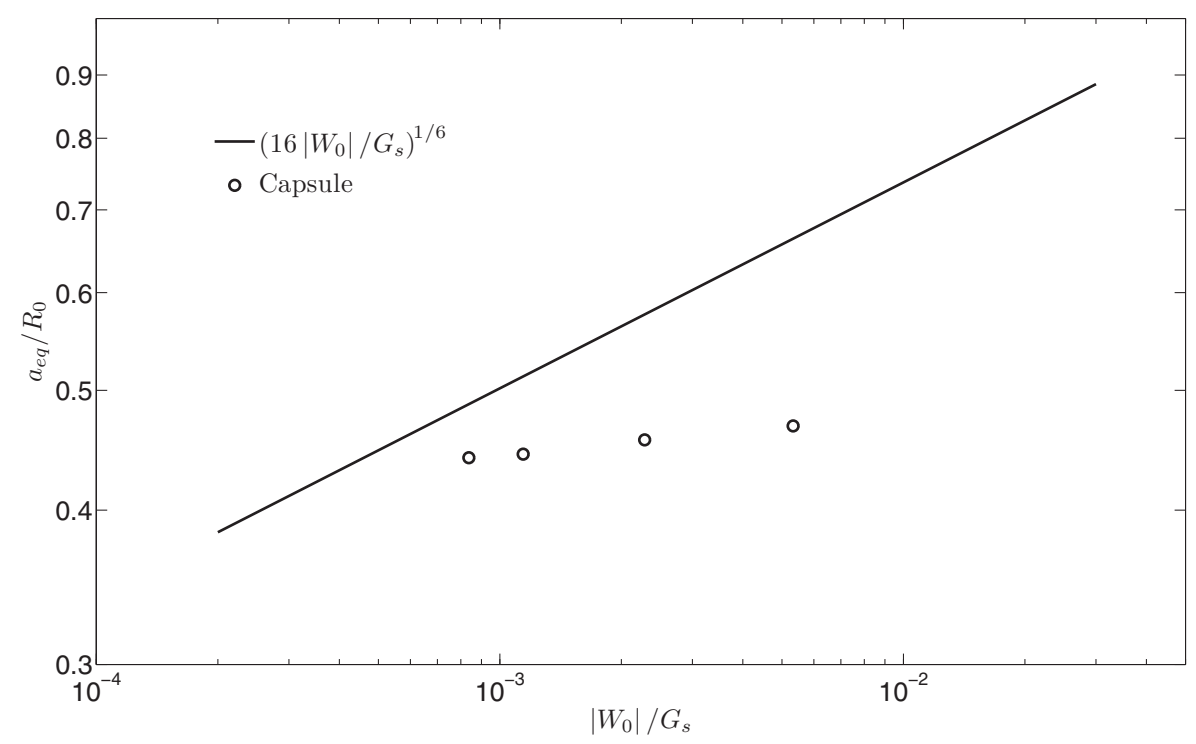

FIG. 6. Dimensionless equilibrium contact radius of capsules versus relative adhesion strength based on the surface shear modulus $\left|W_{0}\right| / G_{s}$ at a fixed value of $\left|W_{0}\right| / K_{s}=7.61 \times 10^{-4}\left(1.1 \leqslant K_{s} / G_{s} \leqslant 7\right)$. 
in past studies $[16,18,27]$ that peeling plays a dominant role throughout the whole detachment process except at the very end when the contribution from pulling becomes significant. However, the condition for the onset of pulling (e.g., whether it occurs at a specific $a / R$ or whether the onset depends on the rate of detachment) is not well understood. It is also unclear whether pulling contributes significantly to the measured dynamic adhesion force. Frostad et al. [16] provided some interesting insight into the hydrodynamic force induced by pulling, based on a parallel disk geometry, and suggested that pulling should play a significant role only at the very end of the process. However, this information is not sufficient to determine the contact radius at the moment when pulling starts to be a significant contributor. For that purpose, we need to compare the minimal force required for peeling and pulling. If we assume that the membrane is moving infinitely slowly so that there is zero viscous dissipation, the minimal force required to reduce the contact radius while holding the surface separation on the line of centers $D_{\text {axis }}$ constant is zero at $a=a_{\mathrm{eq}}$ and increases to $2 \pi R_{0}\left|W_{0}\right|$ as $a \rightarrow 0$. The required force is zero at $a=a_{\text {eq }}$ because the elastic force that is trying to restore the reference shape of the particle balances the surface forces. As the contact radius decreases, the help from elasticity diminishes rapidly according to Eqs. (23) and (26). With the same infinitely slow membrane movement assumption, the minimal force required to separate the two surfaces at constant $a$ scales as $\left(\left|W_{0}\right| / D_{0}\right) \pi a^{2}$. At the very beginning, peeling always dominates the detachment process (zero force required), but as $a$ decreases from $a_{\mathrm{eq}}$, the ratio

$$
\frac{F_{\text {peeling }}}{F_{\text {pulling }}}=\frac{2 \pi R_{0}\left|W_{0}\right|}{\left(\left|W_{0}\right| / D_{0}\right) \pi a^{2}}=\frac{2 D_{0} R_{0}}{a^{2}}
$$

can help shed some light on whether peeling or pulling is the dominating detachment process for both vesicles and capsules. Since $D_{0} \ll a_{\mathrm{eq}}<R_{0}$ is always true in the cases that we are considering (micron scale particles and surface forces with a range of nanometers), peeling requires less force until $a$ has significantly decreased from $a_{\text {eq. }}$. Pulling starts to be part of the picture when the left-hand side of Eq. (27) become close to unity as $a / R_{0}$ reduces to $a^{*} / R_{0}=\sqrt{2 D_{0} / R_{0}}$.

In simulations, we investigate the detachment process by applying a far-field uniform flow in an attempt to remove the adhered capsule, which is in equilibrium with the rigid surface before the flow is turned on (see Fig. 1). In all the cases that we have investigated numerically $(0.000761 \leqslant$ $\left|W_{0}\right| / K_{s} \leqslant 0.071$ and $0.00676 \leqslant D_{0} / R_{0} \leqslant 0.021$ ), peeling is shown to be the dominant mechanism for most of the detachment process as expected. Two representative cases are presented in Figs. 7(a) and 7(b). Although it is hard to define an exact value of $a$ at which pulling starts to play a role, it is generally shown in the figures that pulling only becomes significant around our estimated transition point $a^{*} / a_{\text {eq }}$. In Fig. 7 (a) there is a sharp dip in $D_{\text {axis }} / D_{0}$ before the two surfaces actually start to pull apart. This is a hydrodynamic effect of the suction pressure caused by two parallel surfaces moving away from each other with a thin layer of fluid in between (the opposite of lubrication pressure) and will be less significant as the difference between the capillary number $\mathrm{Ca}$ and the critical value $\mathrm{Ca}_{c}$, which is the minimum Ca to fully remove the adhered particle from the rigid surface, is reduced, as in Fig. 7(b). The critical force required for detachment without any viscous dissipation was previously reported to be proportional to $R_{0}$ and $\left|W_{0}\right|[13,18]$ and for a spherical vesicle or capsule leaving a rigid flat surface $F_{\text {critical }}=2 \pi R_{0}\left|W_{0}\right|$. The force exerted on the adhered particle by the flow is

$$
F_{\text {hydro }}=k_{1} \mu u_{\infty} R_{0},
$$

where $k_{1}$ is a coefficient largely determined by the geometry of the boundaries. The critical capillary number $\mathrm{Ca}_{c}$ can be found by equating $F_{\text {critical }}$ and $F_{\text {hydro }}$ and dividing both sides by $K_{s}$,

$$
\mathrm{Ca}_{c}=\frac{\mu u_{\infty}}{K_{s}}=\frac{2 \pi}{k_{1}} \frac{\left|W_{0}\right|}{K_{s}} \sim \frac{\left|W_{0}\right|}{K_{s}} .
$$

In simulations, however, the critical value cannot be obtained exactly since as $\mathrm{Ca} \rightarrow \mathrm{Ca}_{c}$, the detachment process becomes infinitely slow. Instead, we establish upper and lower bounds for $\mathrm{Ca}_{c}$ between fully detached cases $\left(\mathrm{Ca}>\mathrm{Ca}_{c}\right)$ and cases where the capsule remains attached $\left(\mathrm{Ca}<\mathrm{Ca}_{c}\right)$ 

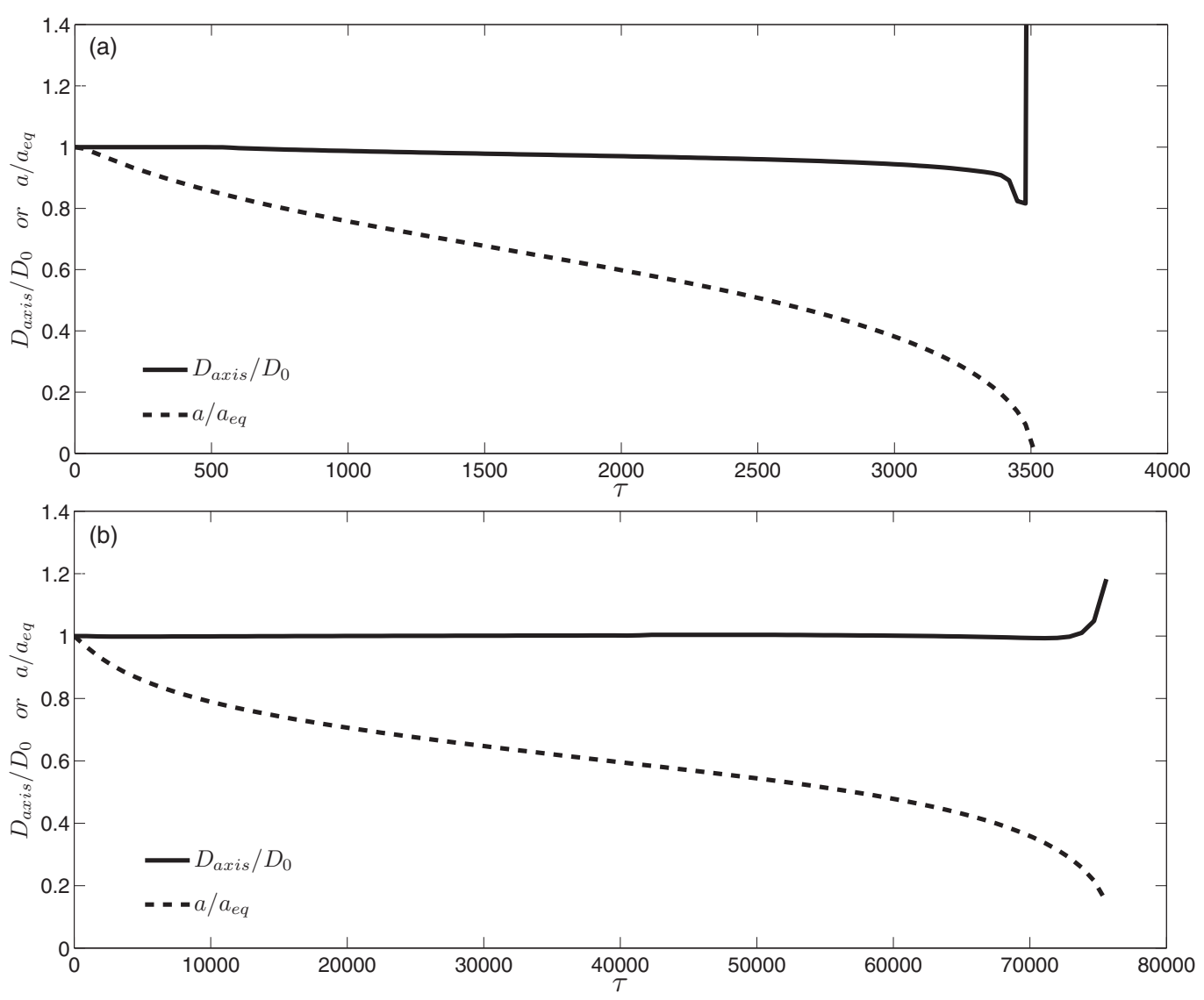

FIG. 7. Simulation results of normalized surface separation on the line of centers $D_{\text {axis }} / D_{0}$ and normalized contact radius $a / a_{\mathrm{eq}}$ versus time. At $\tau=0$, the capsule is in equilibrium with the rigid wall and the far field flow is turned on. The parameters are (a) $a_{\mathrm{eq}} / R_{0}=0.732, D_{0} / R_{0}=0.00676, \mathrm{Ca}=0.5$, and $a^{*} / a_{\mathrm{eq}}=0.158$ and (b) $a_{\mathrm{eq}} / R_{0}=0.456, D_{0} / R_{0}=0.021, \mathrm{Ca}=0.023$, and $a^{*} / a_{\mathrm{eq}}=0.205$.

as shown in Fig. 8. The linear scaling between $\mathrm{Ca}_{c}$ and $\left|W_{0}\right| / K_{s}$ is consistent with what we have obtained from simulation with a fitting parameter of $2 \pi / k_{1}=22$.

\section{Peeling dynamics: Constant far-field flow}

For the peeling process, there is a classical theory developed by Brochard-Wyart and de Gennes [13] for vesicles. However, there are some major limitations in their theory. First of all, they assume a constant and uniform membrane tension. This only makes sense for vesicles that are partially sucked into a pipette since the membrane tension is set by the suction pressure and the tension on a vesicle membrane is close to uniform because there is no shear modulus. This assumption falls apart if detachment is induced by an external flow or body forces like gravity without the pipette. The membrane tension decreases, rather than remaining constant, as the contact radius reduces. Furthermore, if the adhered particle is a capsule instead of a vesicle, the presence of a nonzero shear modulus invalidates the uniform and constant tension assumption. Second, the viscous dissipation term in their theory is adopted from an earlier work on two-dimensional liquid wetting and spreading on a surface [27]. This basically excludes the situation where there is a thin liquid film between the two surfaces, which is quite often the case for capsules, vesicles, or biological cells. 


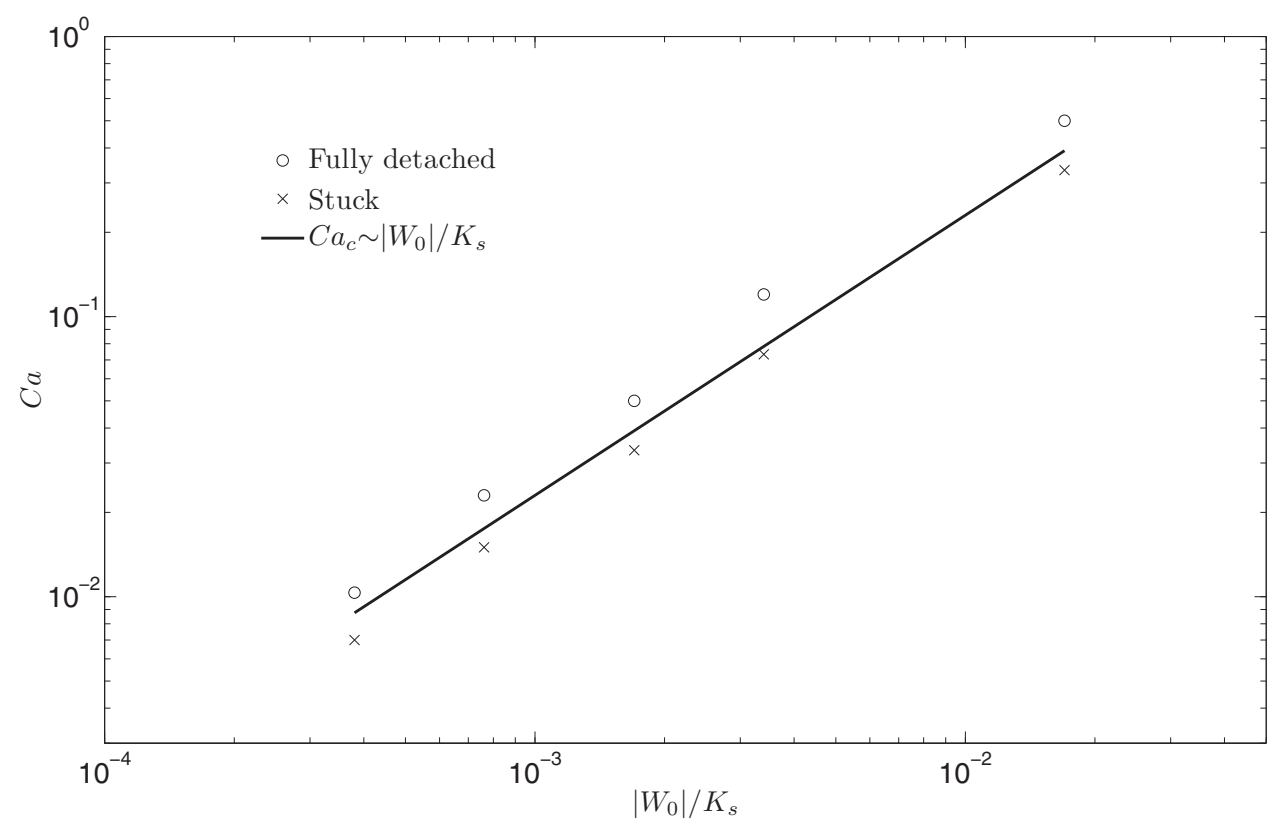

FIG. 8. Critical capillary number $\mathrm{Ca}_{c}$ of adhered capsules bracketed by fully detached cases and stuck cases at various values of $\left|W_{0}\right| / K_{s}$. The solid line is Eq. (29) fitted with $2 \pi / k_{1}=22$ to help visualize the linear relationship between $\mathrm{Ca}_{c}$ and $\left|W_{0}\right| / K_{s}$.

In this work we propose a more general theory for the peeling process of membrane bound particles based on conservation of energy:

$$
\dot{\omega}_{\text {hydro }}=\dot{U}_{\text {interaction }}+\dot{U}_{\text {elastic }}+\Phi,
$$

where $\dot{\omega}_{\text {hydro }}=F_{\text {hydro }} \cdot v_{\text {center }}$ is the rate of work done by the hydrodynamic force [see Eq. (28)] exerted on the particle, with $v_{\text {center }}$ the velocity of the center of mass of the adhered particle, $\dot{U}_{\text {inter }}$ is the rate of change of the surface interaction energy, $\dot{U}_{\text {elas }}$ is the rate of change of the membrane elastic energy, and $\Phi$ is the viscous dissipation in the suspending fluid. A schematic sketch of the geometry of the adhered particle is given in Fig. 9. If we assume that peeling dominates (constant surface separation $D$ ) and the particle maintains a truncated sphere geometry (no elongation) during peeling, the only remaining component of $v_{\text {center }}$ is the diminishing length of the spherical cap $d \approx a^{2} / 2 R_{0}$ (by geometry [18]) during peeling

$$
v_{\text {center }} \approx-\frac{\partial d}{\partial t} \approx-\frac{a}{R_{0}} \frac{\partial a}{\partial t} .
$$

Further, $\dot{U}_{\text {interaction }}$ and $\dot{U}_{\text {elastic }}$ are simply the time derivatives of Eqs. (22) and (23), respectively, and the only time-dependent quantity is the contact radius as shown below:

$$
\begin{gathered}
\dot{U}_{\text {interaction }}=-2 \pi\left|W_{0}\right| a \dot{a}, \\
\dot{U}_{\text {elastic }}=c_{1} \frac{\pi}{8} K_{s}\left(\frac{a}{R_{0}}\right)^{7} R_{0} \dot{a},
\end{gathered}
$$

with $\dot{a}=\partial a / \partial t$. The constant $c_{1}$ is an order one correction coefficient for ignoring the shear modulus and it is equal to 1.4 for cases with $K_{s} / G_{s}=3$ as shown earlier. Finally, viscous dissipation, which 


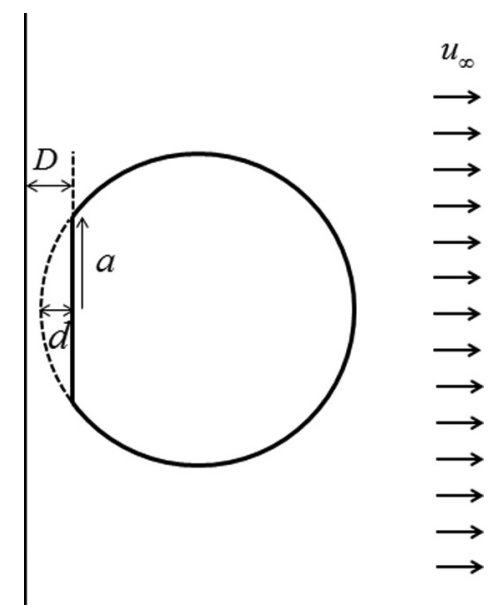

FIG. 9. Schematic sketch of the geometry of the adhered particle under flow. The shape of the particle is approximated by a truncated sphere. During the peeling process, $D$ holds constant while $a$ and $d$ decrease over time.

is concentrated near the edge of the contact zone, can be approximated as $[15,16]$

$$
\Phi=k_{2} \frac{2 \pi \mu a^{2} \dot{a}^{2}}{D_{0}}
$$

where $k_{2}$ is an integration constant. Now we can plug Eqs. (28) and (31)-(34) into Eq. (30):

$$
k_{1} \mu u_{\infty} R_{0}\left(-\frac{a}{R_{0}} \dot{a}\right)=-2 \pi\left|W_{0}\right| a \dot{a}+c_{1} \frac{\pi}{8} K_{s}\left(\frac{a}{R_{0}}\right)^{7} R_{0} \dot{a}+k_{2} \frac{2 \pi \mu a^{2} \dot{a}^{2}}{D_{0}},
$$

and rearrange to obtain an expression for the nondimensional peeling velocity

$$
\frac{\dot{a} \mu}{K_{s}}=\frac{1}{k_{2}} \frac{D_{0}}{R_{0}}\left[-\frac{k_{1}}{2 \pi} \frac{R_{0}}{a} \mathrm{Ca}+\frac{R_{0}}{a} \frac{\left|W_{0}\right|}{K_{s}}-c_{1} \frac{1}{16}\left(\frac{a}{R_{0}}\right)^{5}\right] .
$$

The terms from left to right originate from viscous dissipation, hydrodynamic force on the particle, nonhydrodynamic surface interactions, and elasticity, respectively. The coefficient $2 \pi / k_{1} \approx 22$ and $c_{1} \approx 1.4$ has already been found by simulation in Sec. III B for the two representative cases.

Dimensionless peeling velocity versus contact radius and contact radius versus time, obtained from simulation of two representative cases, are plotted in Figs. 10 and 11, respectively. Equation (36) and the radius obtained by integrating Eq. (36) are also plotted in Figs. 10 and 11, respectively, with one coefficient $k_{2}$ fitted for each set of cases. Both Figs. 10 and 11 show that Eq. (36) predicts the peeling velocity and the time evolution of the contact radius well despite the fact that there is no shear energy term in Eq. (36).

Based upon this result from Eq. (36) (Figs. 10 and 11), the shear modulus seems to have no obvious role in the peeling problem. However, the shear modulus is actually important in terms of maintaining the truncated sphere geometry and thus maintaining a constant value of $c_{1}$. In Fig. 12 two sets of images are produced under almost identical simulation conditions, meaning the same surface interaction and the same Ca based on $K_{s}$, except that the set of Figs. 12(a)-12(d) is a capsule with $K_{s} / G_{s}=3$ and the set of Figs. $12\left(\mathrm{a}^{\prime}\right)-12\left(\mathrm{~b}^{\prime}\right)$ is a vesicle with $G_{s}=0$. It can be seen that a vesicle can be elongated much more easily than a capsule because of the zero shear modulus. This causes the truncated sphere approximation to break down at a lower Ca for vesicles compared to capsules. Once the vesicle or capsule becomes elongated, there is no simple way to estimate the elastic energy and the hydrodynamic force exerted on the particle as in Eqs. (26) and (28), but numerical simulations 

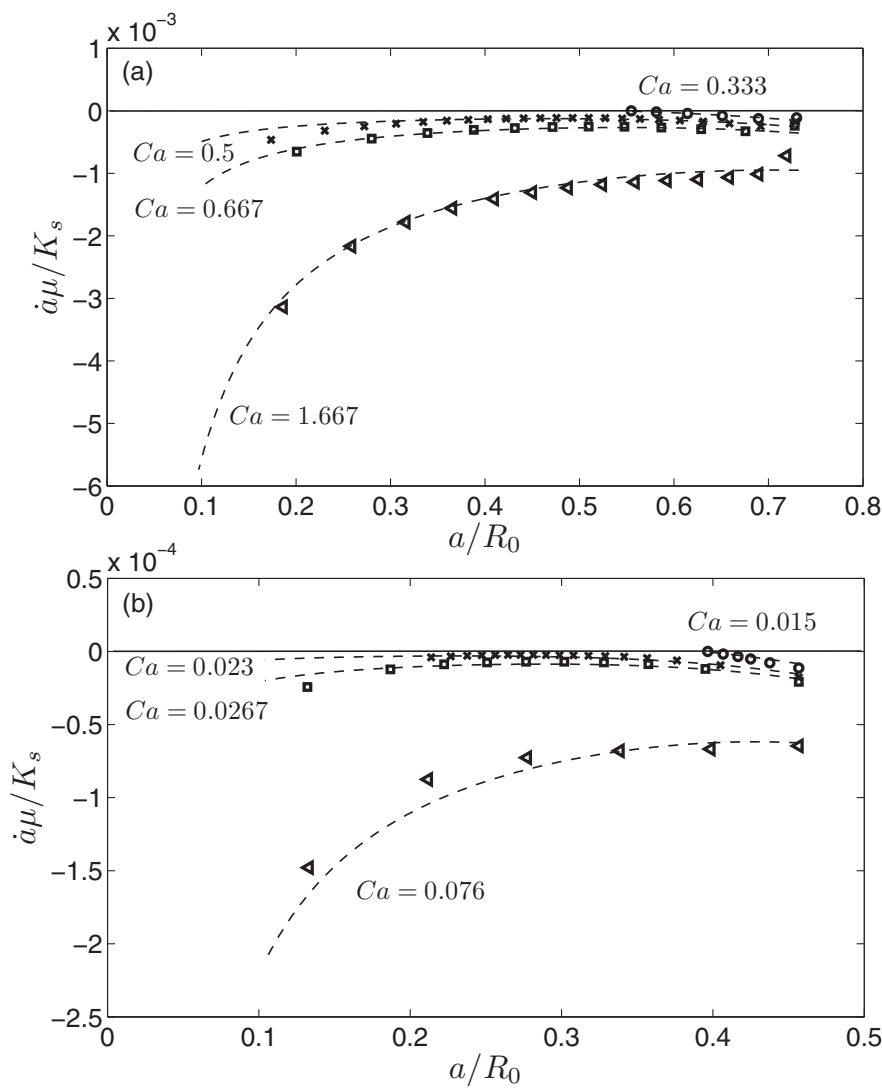

FIG. 10. Dimensionless peeling velocity versus contact radius for capsules with $K_{s} / G_{s}=3$ at various capillary numbers: (a) $k_{2}=0.71, D_{0} / R_{0}=0.00676,\left|W_{0}\right| / K_{s}=0.017$, and $a_{\mathrm{eq}} / R_{0}=0.732$ and (b) $k_{2}=2.36$, $D_{0} / R_{0}=0.021,\left|W_{0}\right| / K_{s}=0.000761$, and $a_{\mathrm{eq}} / R_{0}=0.456$. Dashes lines and various shapes of markers correspond to Eq. (36) and simulation results, respectively.
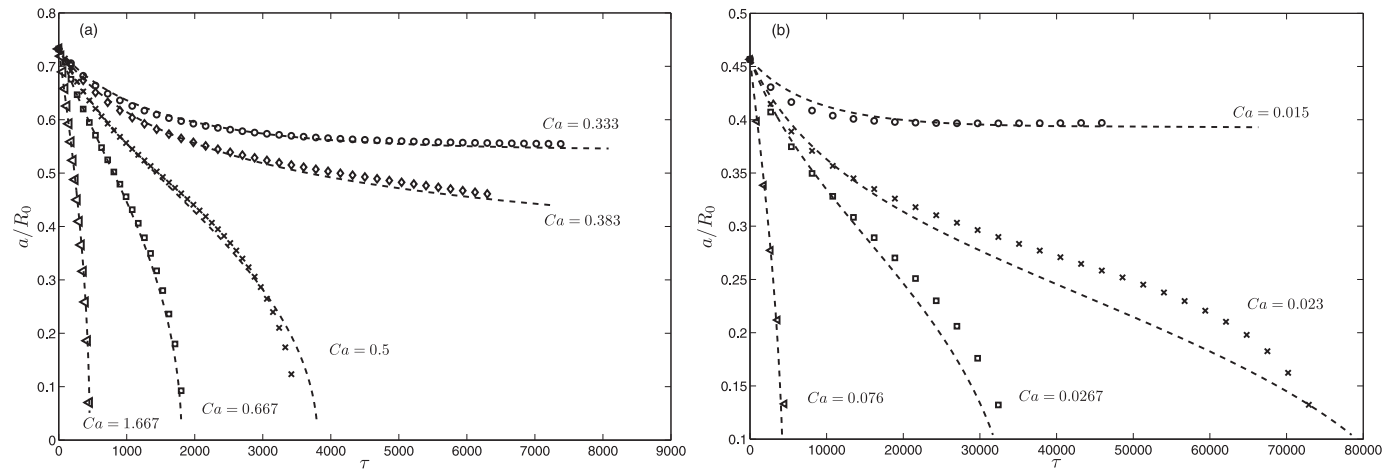

FIG. 11. Dimensionless contact radius versus time for capsules with $K_{s} / G_{s}=3$ at various capillary numbers: (a) $D_{0} / R_{0}=0.00676,\left|W_{0}\right| / K_{s}=0.017$, and $a_{\mathrm{eq}} / R_{0}=0.732$ and (b) $D_{0} / R_{0}=0.021,\left|W_{0}\right| / K_{s}=$ 0.000761 , and $a_{\mathrm{eq}} / R_{0}=0.456$. Dashes lines and various shapes of markers correspond to time integration of Eq. (36) and simulation results, respectively. 

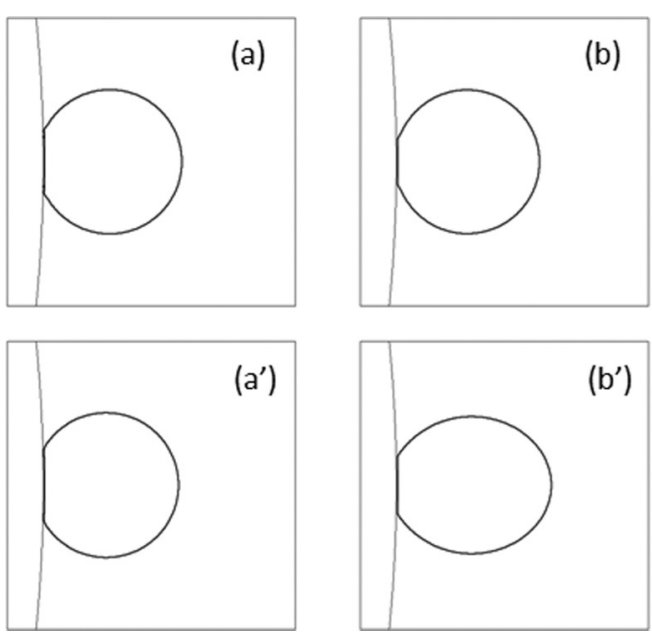

$\left(b^{\prime}\right)$

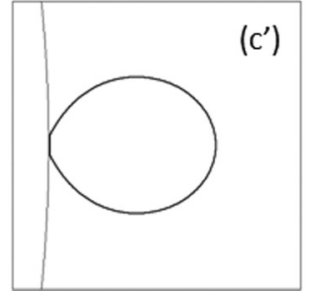

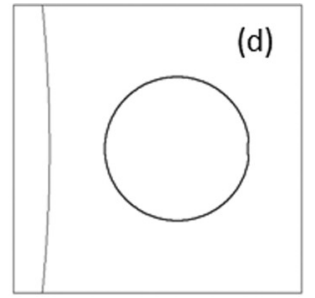

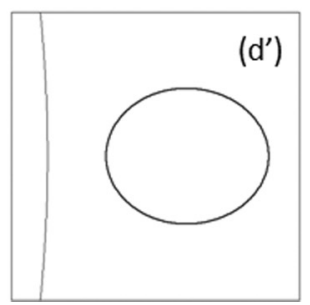

FIG. 12. Shape evolution of a detaching capsule and vesicle at $\mathrm{Ca}=\mu u_{\infty} / K_{s}=0.023, D_{0} / R_{0}=0.021$, and $\left|W_{0}\right| / K_{s}=0.000761:$ (a)-(d) a capsule with $K_{s} / G_{s}=3$ and $\left(\mathrm{a}^{\prime}\right)-\left(\mathrm{d}^{\prime}\right)$ a vesicle with $G_{s}=0$.

can still be relied on to study the problem. Equation (36) is found to be valid for capsules with $K_{s} / G_{s}=3$ up to $\mathrm{Ca}=O(1)$, but for vesicles it only works up to $\mathrm{Ca}=O\left(10^{-4}\right)$. Furthermore, it can be seen by comparing Figs. 13 and 11 that the contact radius decreases much faster under the same flow strength and the critical $\mathrm{Ca}$ is also lower under the same adhesion strength for a vesicle. The elongation extends the vesicle away from the collector. As a result, the surface interaction is weakened and the hydrodynamic force exerted on the particle is increased because the local flow becomes stronger away from the stationary collector. Both of these effects cause the critical Ca to decrease and the peeling velocity to increase. Theoretically, Eq. (36) should work perfectly fine for vesicles with $\mathrm{Ca}=O\left(10^{-4}\right)$ or smaller. However, in order to still have full detachment at such a low

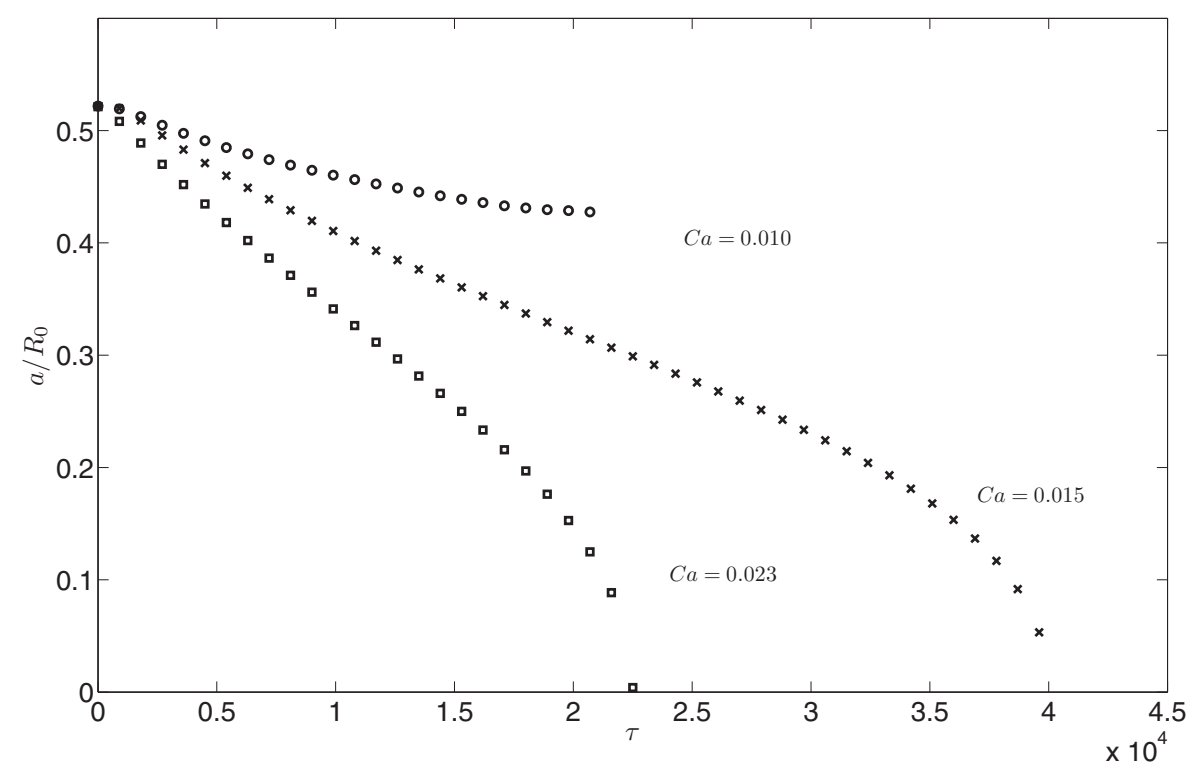

FIG. 13. Dimensionless contact radius versus time for vesicles with $G_{s}=0$ at $D_{0} / R_{0}=0.021,\left|W_{0}\right| / K_{s}=$ $0.000761, a_{\mathrm{eq}} / R_{0}=0.456$, and various capillary numbers. 
capillary number, $\left|W_{0}\right| / K_{s}$ has to be small, according to Eq. (29), which means that the equilibrium contact radius must be small. In this parameter range, the vesicle can be approximated as a solid sphere since there is little deformation due to either surface forces or flow and therefore it is outside the main interest of this study.

\section{Peeling dynamics: Constant peeling velocity}

In the previous section, the discussion of peeling dynamics focused on cases in which peeling is caused by a constant far-field flow. Since the capsules maintain a geometry close to a truncated sphere during peeling, these cases can be considered approximately as peeling with a constant force exerted on the particle. Although in many applications a far-field flow is indeed the most common driving force for detachment, micropipette-based techniques are popular for studying the detachment process simply because they offer more control of the particle and allow measurement of the force applied on the particle throughout the experiments [16,28].

In micropipette detachment experiments, the pipette is often moved away from a second pipette or a substrate at a constant velocity and this leads to a roughly constant peeling velocity $\dot{a}$ [see Fig. 3(b) in Ref. [16]]. Notice that the vesicles are not partially sucked into the pipettes in the experimental work of Frostad et al. [16], so the classical theory of Brochard-Wyart and de Gennes [13] is not applicable here. In the preceding section, the hydrodynamic force on the particle was assumed to be independent of time and the radius $a$ was assumed to vary at a variable rate $\dot{a}$. In the Frostad et al. experiments, $\dot{a}$ is held approximately constant and it is the force that varies with time. In order to compare our theory to the Frostad et al. vesicle experiments at least qualitatively, Eq. (30) is rewritten in the form of the external force $F_{\text {ext }}$ exerted on the particle by either the pipette or the flow as a function of $\dot{a}$ without the flow

$$
\frac{F_{\text {ext }}}{K_{s} R_{0}}=-2 \pi \frac{\left|W_{0}\right|}{K_{s}}+\frac{\pi}{8}\left(\frac{a}{R_{0}}\right)^{6}+\frac{a}{D_{0}} \frac{\mu \dot{a}}{K_{s}},
$$

with coefficients $c_{1}$ and $k_{2}$ set to unity. Equation (37) is plotted in Fig. 14 with the characteristic parameters of Frostad et al. [16], $\left|W_{0}\right|=0.083 \mathrm{~mJ} \mathrm{~m}^{-2}, K_{s}=100 \mathrm{~mJ} \mathrm{~m}^{-2}, D_{0}=5 \mathrm{~nm}$, and $R_{0}=40 \mu \mathrm{m}$, at various dimensionless peeling velocities $\mu \dot{a} / K_{s}$. For infinitely slow peeling $\left(\mu \dot{a} / K_{s}=0\right)$, the force curve intercepts the $x$ axis at $a / R_{0}=a_{\mathrm{eq}} / R_{0}=0.486$ since the surface interaction term and the elasticity term cancel each other and there is no dissipation. For $a / R_{0}>a_{\mathrm{eq}} / R_{0}$, the elasticity of the particle tends to reduce the contact radius and a compressive external force $\left(F_{\text {ext }}>0\right)$ would be required to maintain the contact radius. For $a / R_{0}<a_{\text {eq }} / R_{0}$, a tensile external force $\left(F_{\text {ext }}<0\right)$ would be required to overcome the attractive surface interaction. The external force becomes more negative as the contact radius reduces since the help from elasticity decreases sharply as the contact radius to the sixth power and reaches a minimum at $a / R=0$. At larger values of the peeling velocity, the intercept with the $x$ axis and the location of the force minimum shift in the direction of higher contact radius (to the left in Fig. 14) and the magnitude of the force minimum increases due to larger viscous dissipation. Notice that Eq. (37) is pure peeling, so it is valid before pulling becomes significant as long as $a / R_{0}>a^{*} / R_{0}$. At $a / R_{0} \approx a^{*} / R_{0}$, the external force is expected to jump to zero as two surfaces quickly separate, which is indicated using the arrow in Fig. 14.

The pattern of the force curves agrees with the results obtained by Frostad et al. [16] [Fig. 8(a)]. We are not trying to compare quantitatively because of the uncertainty in the actual values of $\left|W_{0}\right|$ and $D_{0}$ in the experimental work and also the difference in geometry. In Ref. [16], Frostad et al. suggested that the minimum external force is measured close to the end of the detachment process at $a \approx a^{*}$ and the main contributions are from viscous dissipation due to pulling and nonhydrodynamic surface forces. However, membrane elasticity was not considered in their force analysis and both their experimental data and our theory suggest that the minimum occurs at $a>a^{*}$ for dynamic peeling $(\dot{a} \neq 0)$, which means that pulling should not be contributing to the magnitude of the force minimum. According to Eq. (37) and Fig. 14, surface forces, membrane elasticity, and viscous 


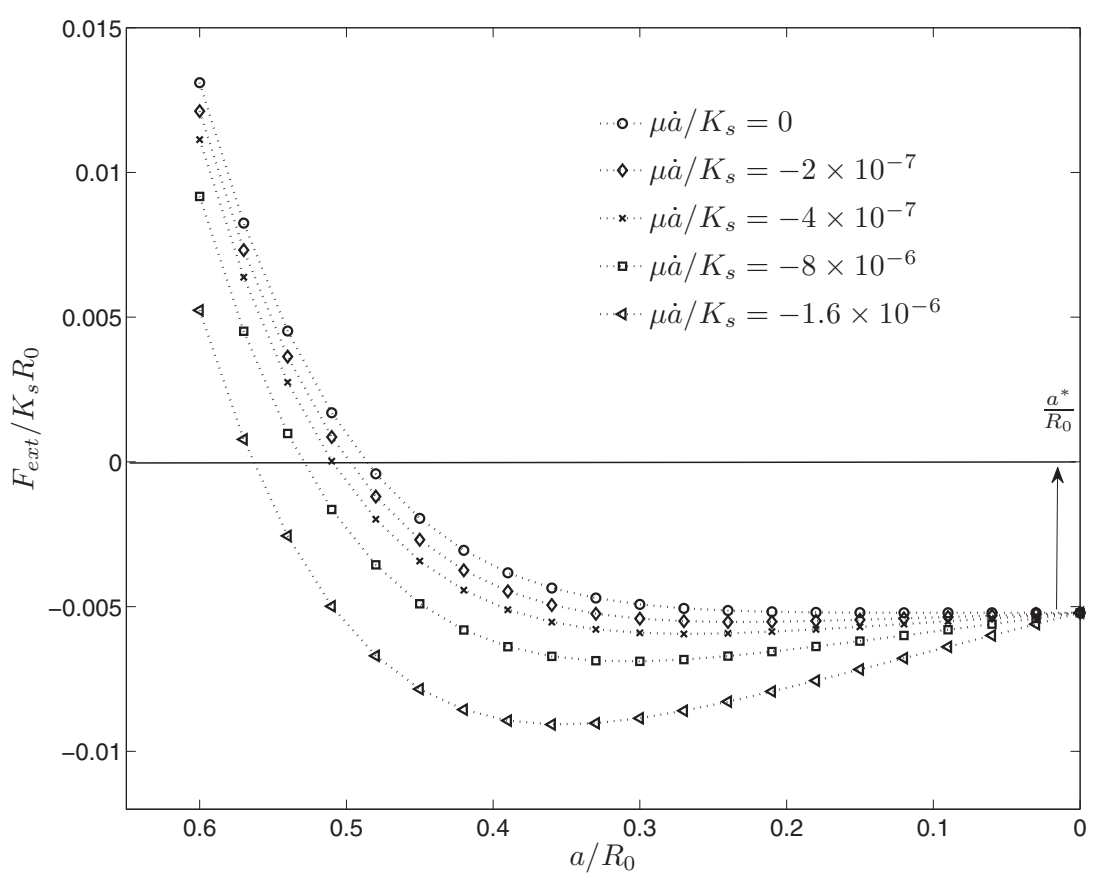

FIG. 14. Dimensionless external force as a function of dimensionless contact area obtained from Eq. (37) with the same parameters as in the experimental work of Frostad et al. [16], $\left|W_{0}\right|=0.083 \mathrm{~mJ} \mathrm{~m}^{-2}$, $K_{s}=100 \mathrm{~mJ} \mathrm{~m}^{-2}, D_{0}=5 \mathrm{~nm}$, and $R_{0}=40 \mu \mathrm{m}$.

dissipation caused by peeling are sufficient to describe the qualitative behavior of the measured force curve for the constant peeling velocity detachment of Frostad et al.

\section{E. Detachment starting from nonequilibrium configurations}

So far we have been mainly focused on cases starting from the equilibrium configuration. Although the equilibrium configuration is an obvious and practical choice as the initial condition for a theoretical study of the detachment problem, in applications, the flow may reverse and detachment may begin before the capsule reaches this equilibrium state. To study this branch of detachment problems numerically, we use a flow (approaching flow) to bring the capsule towards the collector

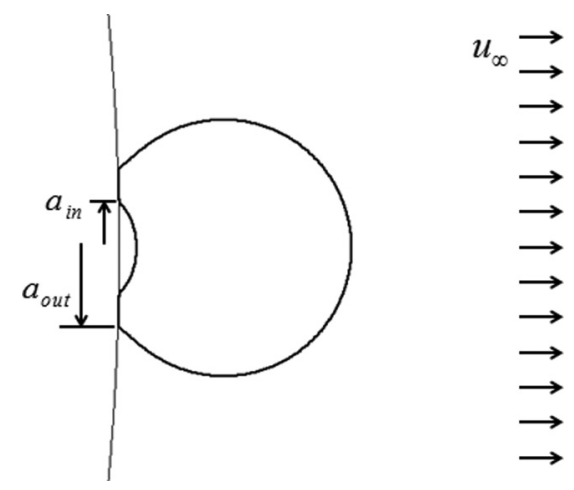

FIG. 15. Typical geometry of an adhered capsule that is subjected to an external flow prior to achieving an equilibrium detachment configuration (when $a_{\mathrm{out}}=a_{\mathrm{eq}}$ and $a_{\mathrm{in}}=0$ ). 

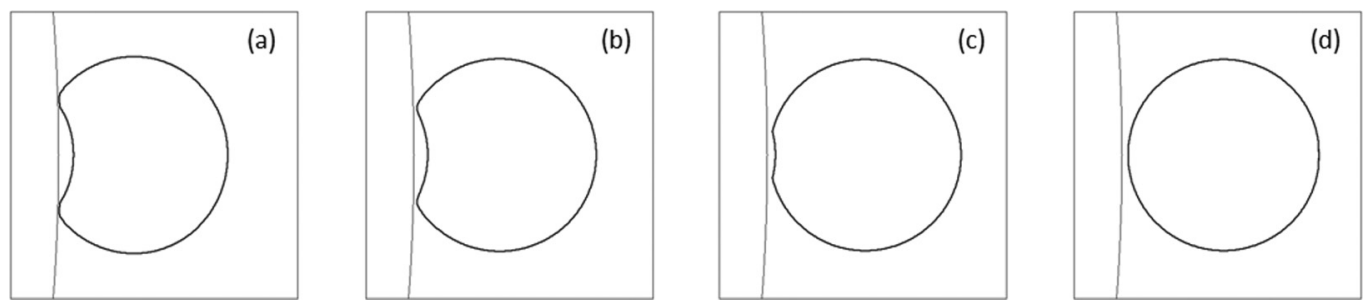

FIG. 16. Spontaneous detachment of an adhered capsule. The approaching flow is replaced by a weak detachment flow $\mathrm{Ca}=0.0002 \ll \mathrm{Ca}_{c} \approx 0.023$ at $a_{\text {in }} / R_{0}=0.52$. The equilibrium configuration is $a_{\text {eq }} / R_{0}=0.456$ with $D_{0} / R_{0}=0.021$ and $\left|W_{0}\right| / K_{s}=0.000761$.

and into the range of surface interactions and then reverse the flow direction in an attempt to detach the capsule (detachment flow) before the capsule has reached the equilibrium state of attachment, as schematically illustrated in Fig. 15. We will show that the critical capillary number for detachment $\mathrm{Ca}_{c}^{*}$ of these nonequilibrium cases either is approximately equal to the equilibrium value $\mathrm{Ca}_{c}^{*} \approx \mathrm{Ca}_{c}$ or is equal to zero $\mathrm{Ca}_{c}^{*}=0$. This means that the minimum external force required to fully detach the adhered capsule is either the same as the equilibrium value or zero (the capsule detaches spontaneously even without any external flow). In particular, there is no partial reduction of the critical detachment capillary number as one might expect.

In numerical simulation, we observed that an adhered capsule with a large inner contact radius $a_{\text {in }}$ resulting from a strong approaching flow and a short allowed period of time for drainage is prone to spontaneous detachment as soon as the approaching flow is removed (i.e., even without any detachment flow). An adhered capsule that undergoes spontaneous detachment is shown in Fig. 16. With a larger $a_{\text {in }}$ at a given $a_{\text {out }}$, there is more deformation and less adhesive contact area and therefore membrane elasticity is likely to dominate over the surface interactions and this leads to spontaneous detachment. Reducing the strength of the approaching flow or maintaining the approaching flow for a longer period of time to reduce $a_{\text {in }}$ below a critical value $a_{c}^{*}$ prevents spontaneous detachment. Note that the cases we investigated in Sec. III A are equivalent to capsules being carried toward the collector with an infinitely slow approaching flow. If the approaching flow is removed at a point
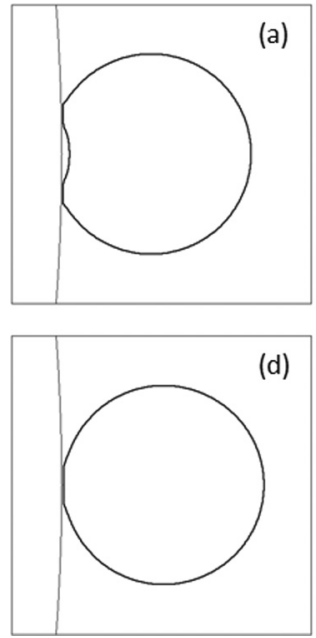
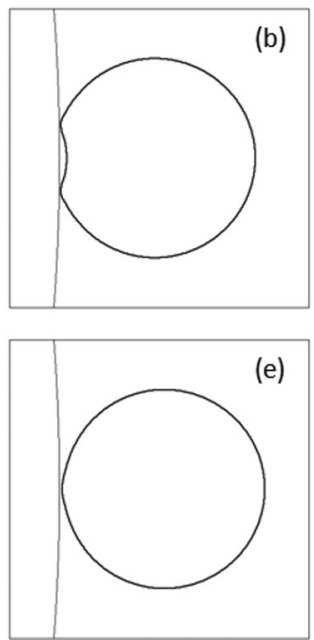
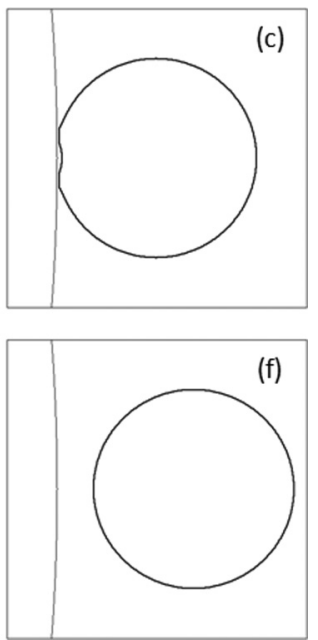

FIG. 17. Detachment of an adhered capsule with trapped fluid in the contact region. The approaching flow is replaced by detachment flow $\mathrm{Ca}=0.023>\mathrm{Ca}_{c} \approx 0.021$ at $a_{\text {in }} / R_{0}=0.31$. The equilibrium configuration is $a_{\text {eq }} / R_{0}=0.456$ with $D_{0} / R_{0}=0.021$ and $\left|W_{0}\right| / K_{s}=0.000761$. 

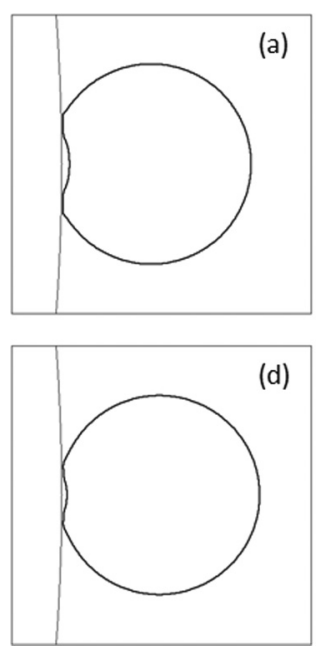
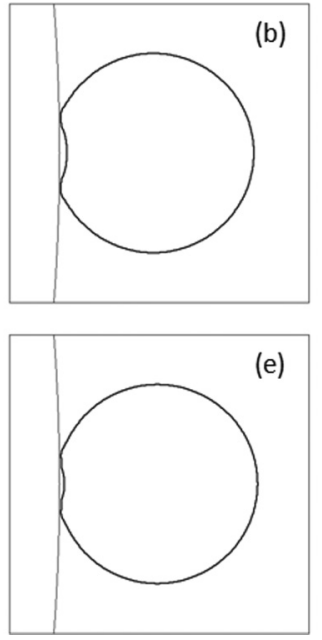
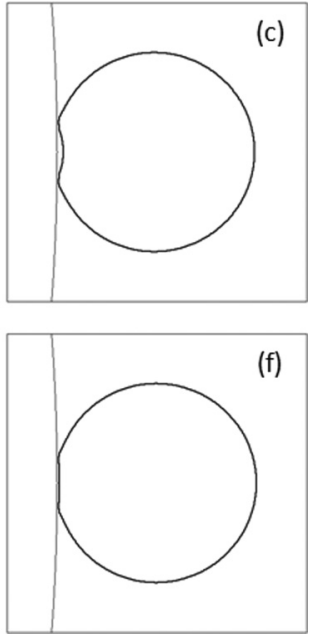

FIG. 18. Adhered capsule with trapped fluid in the contact region initially stays adhered under a weak detachment flow $\mathrm{Ca}=0.02<\mathrm{Ca}_{c} \approx 0.021$. The approaching flow is replaced by detachment flow at $a_{\text {in }} / R_{0}=$ 0.31 . The equilibrium configuration is $a_{\mathrm{eq}} / R_{0}=0.456$ with $D_{0} / R_{0}=0.021$ and $\left|W_{0}\right| / K_{s}=0.000761$.

of time where $a_{\mathrm{in}}<a_{c}^{*}$, the adhered capsule will go to the equilibrium configuration over time ( $a_{\text {out }}=a_{\text {eq }}$ and $a_{\text {in }}=0$ ). Furthermore, if the detachment flow replaces the approaching flow or starts at any point of time with $a_{\text {in }}<a_{c}^{*}$, the flow strength needs to be as strong as in the equilibrium cases $\mathrm{Ca}_{c}$ to fully detach the capsule (for reasons that are explained below).

Under a strong enough $\left(\mathrm{Ca}>\mathrm{Ca}_{c}^{*}\right)$ detachment flow, the outer contact radius $a_{\text {out }}$ decreases due to peeling while $a_{\text {in }}$ decreases due to drainage as shown in Fig. 17. Since the peeling velocity is infinitely slow at $\mathrm{Ca}=\mathrm{Ca}_{c}^{*}$ by definition, the trapped fluid will always be fully drained before the detachment process completes. Hence, the last moment of detachment is the same for both nonequilibrium and equilibrium cases and therefore $\mathrm{Ca}_{c}^{*}=\mathrm{Ca}_{c}$. If $\mathrm{Ca}<\mathrm{Ca}_{c}^{*}$, the adhered capsule will remain partially attached and $a_{\text {in }}$ vanishes over time as shown in Fig. 18. If we compare a case where the capsule becomes detached from one where it does not, we can see that the critical question is whether $a_{\text {out }}$ stops decreasing before it reaches the initial value of $a_{\text {in }}$. Let us denote the point where $a_{\text {out }}$ stops moving inward by $a_{c}^{*}$. Then if $a_{c}^{*}$ is larger than the initial value of $a_{\text {in }}$, the capsule will remain attached, all of the trapped fluid will eventually escape (i.e., $a_{\text {in }} \rightarrow 0$ ), and $a_{\text {out }}$ may make some further minor adjustment ( $a_{\text {out }} \rightarrow a_{\text {eq }}$ if there were no flow). On the other hand, if $a_{c}^{*}$ is smaller than the initial value of $a_{\text {in }}$, the capsule is prone to detach even if there were no flow.

An estimation of $a_{c}^{*}$ can be derived by minimization of the sum of the interaction energy and the elastic energy at a fixed $a_{\text {in }}$ without any external flow:

$$
\frac{d}{d a_{\text {out }}}\left(U_{\text {interaction }}+U_{\text {elastic }}\right)=0 \quad \text { at } a_{\text {out }}=a_{c}^{*},
$$

where

$$
U_{\text {interaction }} \approx-\left|W_{0}\right| \pi\left(a_{\text {out }}^{2}-a_{\text {in }}^{2}\right)
$$

and

$$
U_{\text {elastic }}=U_{\text {area }}=K_{s} \frac{\left(A-A_{0}\right)^{2}}{A_{0}} \approx \frac{1}{256} K_{s}\left(\frac{a_{\text {out }}^{4}}{R_{0}^{4}}+3 \frac{a_{\mathrm{in}}^{4}}{R_{0}^{4}}\right)^{2} A_{0} .
$$

We assume that $a_{\text {in }}$ is constant to simplify our analysis. The inner contact radius $a_{\text {in }}$ decreases over time with or without an external flow, but it is often very slow compared to the peeling velocity due 

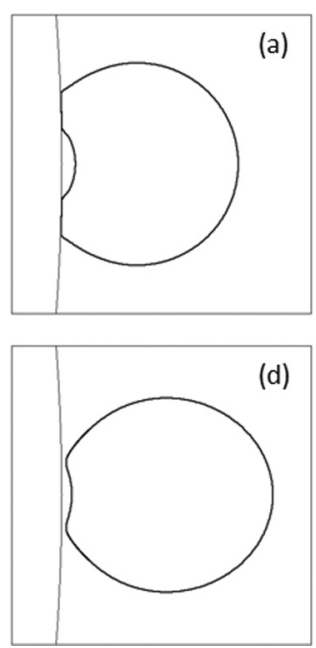

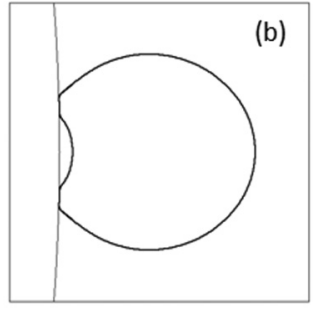

(e)

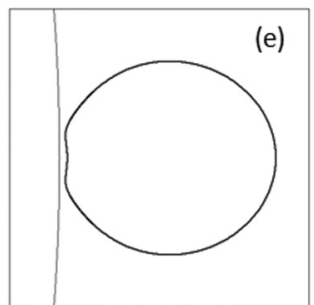

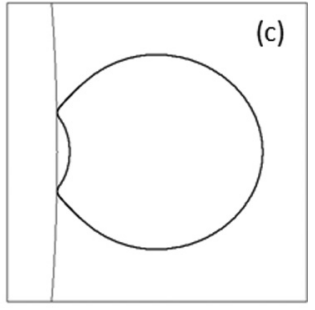

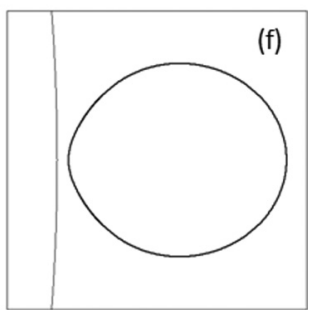

FIG. 19. Detachment of an adhered capsule with trapped fluid in the contact region. The approaching flow is replaced by detachment flow $\mathrm{Ca}=0.5>\mathrm{Ca}_{c} \approx 0.4$ at $a_{\text {in }} / R_{0}=0.29$. The equilibrium configuration is $a_{\text {eq }} / R_{0}=0.732$ with $D_{0} / R_{0}=0.00676$ and $\left|W_{0}\right| / K_{s}=0.017$.

to the thin gap between the membrane and the collector. The resulting expression for $a_{c}^{*}$ is

$$
\left(\frac{a_{c}^{*}}{R_{0}}\right)^{6}+3\left(\frac{a_{c}^{*}}{R_{0}}\right)^{2}\left(\frac{a_{\mathrm{in}}}{R_{0}}\right)^{4}=\frac{16\left|W_{0}\right|}{K_{s}}=\left(\frac{a_{\mathrm{eq}}}{R_{0}}\right)^{6} .
$$

In the limit of small $a_{\text {in }}\left(a_{\text {in }}^{4} \ll a_{\mathrm{eq}}^{4}\right), a_{c}^{*}$ reduces to $a_{\mathrm{eq}}$, which means that, without any external flow, $a_{\text {out }}$ goes to $a_{\mathrm{eq}}$ and $a_{\text {in }}$ goes to zero over time, the adhered capsule goes to the equilibrium configuration. The critical condition for spontaneous detachment is $a_{c}^{*}=a_{\text {in }}$. Plugging the condition into Eq. (41), we obtain $a_{c}^{*}=a_{\text {in }}=0.793 a_{\text {eq }}$. If we further increase the inner contact radius to $a_{\text {in }}=a_{\text {eq }}, a_{c}^{*}$ drops to $0.568 a_{\text {eq }}$. The above calculations imply that when $a_{\text {in }}$ is equal to or smaller than $0.793 a_{\text {eq }}$ spontaneous detachment will not occur and the critical capillary number for detachment is the same as in the equilibrium case $\mathrm{Ca}_{c}^{*}=\mathrm{Ca}_{c}$. For $a_{\text {in }}>0.793 a_{\text {eq }}$, spontaneous detachment $\left(\mathrm{Ca}_{c}^{*}=0\right)$ is contingent upon a slow drainage of the trapped fluid compared to the peeling velocity. Basically, an adhered capsule with large values of $a_{\text {in }} / a_{\text {eq }}>0.793$ and $K_{s} /\left|W_{0}\right|$ (strong elasticity) and small $D_{0} / R_{0}$ (narrow gap and slow drainage) is more likely to experience spontaneous detachment.

If a capillary number that is larger than the critical value is applied $\mathrm{Ca}>\mathrm{Ca}_{c}$ along with a thin gap between the two surfaces $D_{0} / R_{0} \ll 1$, the capsule could leave the collector before all the trapped fluid is drained (i.e., separation occurs at $a_{\text {in }} \neq 0$ ) as shown in Fig. 19. Under such conditions, the outer radius $a_{\text {out }}$ decreases relatively quickly while $a_{\text {in }}$ remains more or less fixed. Although the critical capillary number remains the same in this type of detachment, the time required to fully detach the adhered capsule from the target surface become less. Using Fig. 19(a) as the initial condition, the times required to fully detach the adhered capsules are $\tau=3420$ and 1350 for $\mathrm{Ca}=0.5$ and 0.667 , respectively. The two cases show $11 \%(\mathrm{Ca}=0.5)$ and $25 \%(\mathrm{Ca}=0.667)$ reduction in the time required to complete the detachment process compared to cases that use the equilibrium configuration as the initial condition with the same capillary number as shown in Fig. 11(b).

\section{CONCLUSION}

Scaling theories and a simulation technique that couples the nonhydrodynamic surface forces, membrane mechanics, and Stokes flow have been developed to study the dynamics of adhesion and detachment of capsules. Simulation data for the equilibrium contact radius, the critical detachment 
capillary number, the peeling velocity under constant flow detachment, and the force on the capsule when the peeling velocity is held constant during detachment were compared to scaling theories.

For the equilibrium contact radius $a_{\text {eq }}$, we posited that the shear modulus is only of secondary importance and thus $a_{\mathrm{eq}}$ should scale as the one-sixth power of the relative adhesion strength $\left|W_{0}\right| / K_{s}$, similar to a vesicle. We confirmed this conjecture with simulation data and showed that having a nonzero $G_{s}$ only reduces the prefactor slightly but that the scaling relation based only on $K_{s}$ still holds. We have also shown through both simulation and scaling theory that peeling is the dominant detachment mechanism throughout most of the detachment process, at least for vesicles or capsules with an internal viscosity that is comparable to or smaller than the external fluid viscosity, while pulling only becomes significant near the end around our estimated transition point $a^{*} / R_{0}=\sqrt{2 D_{0} / R_{0}}$. In peeling dynamics, an expression has been derived that can be used to estimate the peeling velocity, or the force on the particle during peeling, based on the fact that $K_{s}$ is the dominating modulus. The results from this expression are consistent with simulation results and experimental results. Ironically, the expression developed by arguing that $G_{s}$ is not important is found to work better for capsules than vesicles in terms of the applicable range of Ca. One would think that it should work better for vesicles, since liquid vesicles have $G_{s}=0$ by definition. However, the fact that vesicles have a zero shear modulus causes the truncated sphere geometry to break down at a lower Ca compared to capsules. Once the truncated sphere geometry is not applicable, it is hard to relate the contact radius to the area strain by a simple relation and only numerical simulation can be used to study the problem.

For the entire problem, we found that the capsule membrane can be described using one material coefficient, the area dilation modulus $K_{s}$, instead of requiring both $K_{s}$ and the shear modulus $G_{s}$, or just $G_{s}$. This is a significant result from the point of view of a general understanding of capsule dynamics. In most other flow problems such as capsule deformation in shear flow or the rheological properties of a capsule suspension, the shear modulus is usually the most relevant modulus and the area dilation modulus is usually of secondary importance [29,30]. These two examples illustrate the important fact that different moduli may control the response of the capsule in different types of deformation and flow. This knowledge is important in designing artificial polymer capsules that mimic biological cells, since $K_{s}$ and $G_{s}$ are usually the same order of magnitude and proportional to one another for capsules (as assumed in the neo-Hookean law for polymeric capsules) while $K_{s}$ is usually one to two orders larger than $G_{s}$ for biological cells with lipid bilayer structures [31,32]. In other words, it is likely that only one modulus can be matched and the best choice depends on whether the adhesion and detachment behavior or the rheological behavior of the biological cell is to be mimicked.

\section{ACKNOWLEDGMENTS}

The authors would like to acknowledge support from the Center for Scientific Computing at the CNSI and MRL through National Science Foundation (NSF) MRSEC (Grant No. DMR-1121053), NSF Grant No. CNS-0960316, and Hewlett Packard and especially Procter \& Gamble for funding this project. The authors would also like to thank Sumanth Jamadagni (Procter \& Gamble) for consultation.

[1] C. Pozrikidis, Numerical simulation of cell motion in tube flow, Ann. Biomed. Eng. 33, 165 (2005).

[2] S. Jadhav, C. D. Eggleton, and K. Konstantopoulos, A 3-D computational model predicts that cell deformation affects selectin-mediated leukocyte rolling, Biophys. J. 88, 96 (2005).

[3] S. K. Doddi and P. Bagchi, Three-dimensional computational modeling of multiple deformable cells flowing in microvessels, Phys. Rev. E 79, 046318 (2009). 
[4] J. Frostad, J. Walter, and L. G. Leal, A scaling relation for the capillary-pressure driven drainage of thin films, Phys. Fluids 25, 052108 (2013).

[5] M. P. Keh, J. Walter, and L. G. Leal, Hydrodynamic interaction between a capsule and a solid boundary in unbounded Stokes flow, Phys. Fluids 26, 111903 (2014).

[6] E. Evans, Analysis of adhesion of large vesicles to surfaces, Biophys. J. 31, 425 (1980).

[7] I. Cantat and C. Misbah, Dynamics and Similarity Laws for Adhering Vesicles in Haptotaxis, Phys. Rev. Lett. 83, 235 (1999).

[8] J. Nam and M. M. Santore, The adhesion kinetics of sticky vesicles in tension: The distinction between spreading and receptor binding, Langmuir 23, 10650 (2007).

[9] A. Ramachandran, T. H. Anderson, L. G. Leal, and J. N. Israelachvili, Adhesive interactions between vesicles in the strong adhesion limit, Langmuir 27, 59 (2011).

[10] K.-T. Wan and K.-K. Liu, Contact mechanics of a thin-walled capsule adhered onto a rigid planar substrate, Med. Biol. Eng. Comput. 39, 605 (2001).

[11] K. Liu, V. Chan, and Z. Zhang, Capsule-substrate contact deformation: Determination of adhesion energy, Med. Biol. Eng. Comput. 40, 491 (2002).

[12] P. Jayathilake, B. Khoo, and Z. Tan, Effect of membrane permeability on capsule substrate adhesion: Computation using immersed interface method, Chem. Eng. Sci. 65, 3567 (2010).

[13] F. Brochard-Wyart and P.-G. de Gennes, Unbinding of adhesive vesicles, C. R. Phys. 4, 281 (2003).

[14] Y. Lin and L. Freund, Forced detachment of a vesicle in adhesive contact with a substrate, Int. J. Solids Struct. 44, 1927 (2007).

[15] S. Chatkaew, M. Georgelin, M. Jaeger, and M. Leonetti, Dynamics of Vesicle Unbinding under Axisymmetric Flow, Phys. Rev. Lett. 103, 248103 (2009).

[16] J. M. Frostad, M. Seth, S. M. Bernasek, and L. G. Leal, Direct measurement of interaction forces between charged multilamellar vesicles, Soft Matter 10, 7769 (2014).

[17] J. Walter, A.-V. Salsac, D. Barthès-Biesel, and P. Le Tallec, Coupling of finite element and boundary integral methods for a capsule in a Stokes flow, Int. J. Numer. Method. Eng. 83, 829 (2010).

[18] J. N. Israelachvili, Intermolecular and Suface Forces (Academic Press, London, 1992).

[19] S. H. Donaldson, C. T. Lee, B. F. Chmelka, and J. N. Israelachvili, General hydrophobic interaction potential for surfactant/lipid bilayers from direct force measurements between light-modulated bilayers, Proc. Natl. Acad. Sci. USA 108, 15699 (2011).

[20] H. Brenner, The slow motion of a sphere through a viscous fluid towards a plane surface, Chem. Eng. Sci. 16, 242 (1961).

[21] S. L. Goren and M. E. O'Neill, On the hydrodynamic resistance to a particle of a dilute suspension when in the neighbourhood of a large obstacle, Chem. Eng. Sci. 26, 325 (1971).

[22] C. Pozrikidis, Boundary Integral and Singularity Methods for Linearized Viscous Flow (Cambridge University Press, Cambridge, 1992).

[23] D. Barthès-Biesel, J. Walter, and A.-V. Salsac, in Computational Hydrodynamics of Capsules and Biological Cells, edited by C. Pozrikidis (CRC Press, Boca Raton, 2010), Chap. 2, pp. 35-70.

[24] R. Skalak, A. Tozeren, R. Zarda, and S. Chien, Strain energy function of red blood cell membranes, Biophys. J. 13, 245 (1973).

[25] C. Quéguiner and D. Barthès-Biesel, Axisymmetric motion of capsules through cylindrical channels, J. Fluid Mech. 348, 349 (1997).

[26] R. Smith, Direct Gauss quadrature formulas for logarithmic singularities on isoparametric elements, Eng. Anal. Bound. Elem. 24, 161 (2000).

[27] P.-G. de Gennes, Wetting: Statics and dynamics, Rev. Mod. Phys. 57, 827 (1985).

[28] M.-J. Colbert, A. Raegen, C. Fradin, and K. Dalnoki-Veress, Adhesion and membrane tension of single vesicles and living cells using a micropipette-based technique, Eur. Phys. J. E 30, 117 (2009).

[29] J. Walter, A.-V. Salsac, and D. Barthès-Biesel, Ellipsoidal capsules in simple shear flow: Prolate versus oblate initial shapes, J. Fluid Mech. 676, 318 (2011).

[30] P. Pranay, R. G. Henriquez-Rivera, and M. D. Graham, Depletion layer formation in suspensions of elastic capsules in Newtonian and viscoelastic fluids, Phys. Fluids 24, 061902 (2012). 
[31] D. Barthès-Biesel, A. Diaz, and E. Dhenin, Effect of constitutive laws for two-dimensional membranes on flow-induced capsule deformation, J. Fluid Mech. 460, 211 (2002).

[32] Y. Tan, D. Sun, W. Huang, and S. H. Cheng, Characterizing mechanical properties of biological cells by microinjection, IEEE Trans. Nanobiosci. 9, 171 (2010). 